\title{
OPERATOR METHODS AND LAGRANGE INVERSION: A UNIFIED APPROACH TO LAGRANGE FORMULAS
}

\author{
CH. KRATTENTHALER
}

\begin{abstract}
We present a general method of proving Lagrange inversion formulas and give new proofs of the s-variable Lagrange-Good formula [13] and the $q$-Lagrange formulas of Garsia [7], Gessel [10], Gessel and Stanton [11, 12] and the author [18]. We also give some $q$-analogues of the Lagrange formula in several variables.
\end{abstract}

1. Introduction. Let $f(z)$ be a formal power series (fps) and $g(z)$ a formal Laurent series (fLs) with finitely many coefficients with negative index different from zero $\left(g(z)=\sum_{k \geq l} g_{k} z^{k}\right.$ for an integer $\left.l\right)$. Let $f(0)=0$ and $f^{\prime}(0) \neq 0$. The coefficients of the expansion $g(z)=\sum_{k \in \mathbf{Z}} c_{k} f^{k}(z)$ can be computed by the two versions of the Lagrange formula, the first of which can be written as

$$
c_{n}=n^{-1}\left\langle z^{-1}\right\rangle g^{\prime}(z) f^{-n}(z) \text { for } n \neq 0, n \in \mathbf{Z} \text { (integers), }
$$

where $\left\langle z^{k}\right\rangle$ means the coefficient of $z^{k}$; the second can be written as

$$
c_{n}=\left\langle z^{0}\right\rangle g(z) \frac{z f^{\prime}(z)}{f^{n+1}(z)} \quad \text { for } n \in \mathbf{Z} .
$$

These formulas are based on the orthogonality relation

$$
\left\langle z^{0}\right\rangle f^{k}(z) \cdot \frac{z f^{\prime}(z)}{f^{n+1}(z)}=\delta_{n k}
$$

for all $n, k \in \mathbf{Z}$ ( $\delta_{n k}$ is the Kronecker delta).

Using Hof bauer's method [16] for an orthogonality relation $\left(f_{k}, \tilde{f}_{n}\right)=\delta_{n k}$ we can transfer certain properties of the sequence $\left(f_{k}\right)_{k \in \mathbf{Z}}$ to the sequence $\left(\tilde{f}_{k}\right)_{k \in \mathbf{Z}}$, where $(,$,$) denotes a bilinear form. Hofbauer used it to prove some one-variable$ Lagrange formulas. We extend this method in $\S 4$ by our Theorems 1 and 5 in order to give a unified "recipe" for proving Lagrange inversion formulas. All known finitedimensional Lagrange formulas can be treated, as we show in $\S \S 5$ to 8 . Moreover we use this recipe to find new Lagrange inversion formulas.

$\S 5$ deals with the Lagrange-Good formula [13]. Using our method, we give a short new proof in which the Jacobian appears in a natural way. We are also able to find multivariable generalizations even of (1.1), the "first version" of the Lagrange formula (identities (5.6) and (5.7)).

Received by the editors November 1, 1985.

1980 Mathematics Subject Classification (1985 Revision). Primary 05A19; Secondary 05A15, $05 \mathrm{~A} 10$.

Key words and phrases. Lagrange inversion formula, $q$-Lagrange inversion formula, inverse relations, umbral operators, $q$-exponential function, $q$-Catalan numbers. 
$\S 6$ contains a new and extended presentation of Garsia's $q$-Lagrange theory [7]. The main idea is to extend his definition of $q$-powers to powers with integral exponents. We show that all of Garsia's results remain true in this more general context. Garsia [7] points out the connection between his theory and Gessel's $q$-Lagrange theorem [10, Theorem 6.9], but he is unable to prove it. Our extension to integral powers together with Theorem 8 , where we discover the connection between Garsia's "right" and "left" inverses, is the key for finding a new proof of Gessel's theorem, within the setting of Garsia's (extended) theory. (Gessel derives it as a special case of a noncommutative generalization of the Lagrange inversion formula.)

In $\S 7$ Gessel and Stanton's [11, 12] $q$-Lagrange formula is discussed. Earlier Carlitz [2] proved an inverse relation which generalizes Gessel and Stanton's formula. We prove both of these results by our operator method and obtain multidimensional generalizations (Theorem 12, Corollary 13).

$\S 8$ concerns our $[18,19] q$-Lagrange formula. In trying to find an $s$-variable $q$-analogue of the Lagrange-Good formula, we succeed only when $s=2$ (Theorems 20,22 and 23), where we find $q$-analogues for special cases of the two-variable Lagrange-Good formula. In Example 21 we give an application to MacMahon's $q$-Catalan numbers.

2. Definitions. Let $\mathbf{Z}$ be the set of integers. For a natural number $s \mathbf{Z}^{s}$ denotes the set of $s$-tuples with the integers as components. For $m=\left(m_{1}, \ldots, m_{s}\right)$ and $n=$ $\left(n_{1}, \ldots, n_{s}\right) \in \mathbf{Z}^{s}$ we set as usual $|m|=\sum_{i=1}^{s} m_{i}, m+n=\left(m_{1}+n_{1}, \ldots, m_{s}+n_{s}\right)$ and $m \leq n$ if and only if $m_{i} \leq n_{i}$ for all integers $i, 1 \leq i \leq s$. If all $m_{i}$ 's are nonnegative $m$ ! means $m_{1} ! \cdots m_{s}$ !. For the special vectors of $\mathbf{Z}^{s}$ where all components are zero except the $i$ th, which is 1 , we write $e_{i}$. The vector $e$ is $(1,1, \ldots, 1)$. For $(0,0, \ldots, 0)$ we simply write 0 . For a set of commuting variables $\left\{z_{1}, z_{2}, \ldots, z_{k}\right\}$ we set $z^{k}=z_{1}^{k_{1}} \cdot z_{2}^{k_{2}} \cdots z_{s}^{k_{s}}$, where $k \in \mathbf{Z}^{s}$.

In this paper sections in which only a single variable is considered are always separated from multivariable sections. Although multi-indices (elements of $\mathbf{Z}^{s}$ ) are not denoted differently than one-dimensional indices (the same is true for variables), no confusion should arise. In the multivariable sections we assume a fixed $s \in \mathbf{N}$ (natural numbers) except in $\S 8$, where $s=2$.

Let $\mathbf{A}$ be a (commutative) integral domain with unity. We shall consider the $\mathbf{A}$ module $\overline{L s}(z)=\overline{L s}\left(z_{1}, \ldots, z_{s}\right)$ of all formal Laurent series having the form $a(z)=$ $\sum_{n \geq k} a_{n} z^{n}$ for some $k \in \mathbf{Z}^{s}$ and $a_{n} \in \mathbf{A}$. Adding elements of $\overline{L s}(z)$ is done by adding the components. The multiplication of $a(z)$ (as above) and $b(z)=$ $\sum_{m \geq l} b_{m} z^{m}$ is defined by

$$
a(z) \cdot b(z)=\sum_{n \geq k+l} \sum_{m} a_{n-m} b_{m} z^{n} .
$$

The inner sum $\sum_{m} a_{n-m} b_{m}$ is finite because of the special form of the elements of $\overline{L s}(z)$; in fact $n-k \geq m \geq l$. Of course $(\overline{L s}(z),+, \cdot)$ is an integral domain.

Next we define some linear operators on $\overline{L s}(z) . L_{0} a(z)$ denotes the coefficient of $z^{0}$ in $a(z)$. The coefficient of $z^{n}$ in $a(z)$ is then $L_{0}\left(z^{-n} \cdot a(z)\right)$, for which we write $\left\langle z^{n}\right\rangle a(z)$. The partial differential operators $D_{i}$ are defined by $D_{i} z^{n}=n_{i} \cdot z^{n-e_{i}}$. Furthermore for the $q$-analogues we need the operators $\varepsilon_{i}^{\left(q_{i}\right)}$ given by $\varepsilon_{i}^{\left(q_{i}\right)} z^{n}=q_{i}^{n_{i}} z^{n}$, where the $q_{i}$ 's are indeterminates. Finally we introduce the partial $q$-difference 
operators by

$$
D_{i}^{\left(q_{i}\right)}=\left[\left(q_{i}-1\right) z_{i}\right]^{-1}\left(\varepsilon_{i}-I\right)
$$

denotes the identity operator). The bilinear form $\langle a(z), b(z)\rangle=L_{0}(a(z) \cdot b(z))$ will be crucially important. For a linear operator $U$ on $\overline{L s}(z)$, the adjoint operator $U^{*}$ is the operator which satisfies $\langle U a(z), b(z)\rangle=\left\langle a(z), U^{*} b(z)\right\rangle$ for all $a(z), b(z) \in \overline{L s}(z)$.

For the indeterminate $q$ we define

$$
\begin{gathered}
(a, q)_{n}=\prod_{i=0}^{n-1}\left(1-a q^{i}\right), \\
{[\alpha]=\frac{q^{\alpha}-1}{q-1}, \quad[n] !=[n] \cdot[n-1] \cdots[1], \quad[0] !=1}
\end{gathered}
$$

and

$$
\left[\begin{array}{l}
\alpha \\
n
\end{array}\right]=\frac{[\alpha] \cdot[\alpha-1] \cdots[\alpha-n+1]}{[n] !}=\frac{\left(q^{\alpha}, q^{-1}\right)_{n}}{(q, q)_{n}}
$$

The $q$-exponential series is

$$
e_{q}(z)=\sum_{k=0}^{\infty} \frac{z^{k}}{[k] !}
$$

Alternative expressions are

$$
e_{q}(z)=\prod_{k=0}^{\infty}\left(1+(q-1) q^{k} z\right)^{-1} \quad \text { and } \quad e_{1 / q}(z)=\prod_{k=0}^{\infty}\left(1+(1-q) q^{k} z\right)
$$

where the infinite products converge as formal power series in $q$, yet may be viewed as formal power series in $z$. For a definition of infinite products of that type as fps in $z$ see [7, p. 217]. In addition $e_{q}(z)^{-1}=e_{1 / q}(-z)$. For an introduction to our notation and $q$-identities see Cigler's paper [3]. Note that in the one-variable sections we write $z$ for $z_{1}, D$ for $D_{1}, D^{(q)}$ for $D_{1}^{\left(q_{1}\right)}$, etc.

3. Preliminaries. We start with a sequence $f=\left(f_{k}(z)\right)_{k \in Z^{s}}$ of elements of $\overline{L s}(z)$ having the form

$$
f_{k}(z)=\sum_{n \geq k} f_{n k} z^{n} \quad \text { and } \quad f_{k k} \text { invertible in } \mathbf{A} .
$$

We call $f$ a diagonal sequence.

Given another diagonal sequence $\mathfrak{g}=\left(g_{l}(z)\right)_{l \in \mathbf{Z}^{s}}$, where $g_{l}(z)=\sum_{k \geq l} g_{k l} z^{k}$, the substitution $\mathfrak{g}(\mathfrak{f})$ is the sequence $\left(h_{l}(z)\right)_{l \in \mathbf{Z}^{s}}$, where

$$
h_{l}(z)=\sum_{k \geq l} g_{k l} f_{k}(z)
$$

or, more precisely,

$$
h_{l}(z)=\sum_{n} \sum_{n \geq k \geq l} f_{n k} g_{k l} z^{n} .
$$

This substitution is associative; the neutral element is the sequence $\underline{1}=\left(z^{n}\right)_{n \in Z^{s}}$. The sequence $\mathfrak{F}=\left(F_{l}(z)\right)_{l \in Z^{s}}$ is called the inverse sequence or short inverse of $\mathfrak{f}$ if $\mathfrak{F}(\mathfrak{f})=\left(z^{n}\right)_{n \in Z^{*}}$. This means that if we set

$$
F_{l}(z)=\sum_{k \geq l} F_{k l} z^{k}
$$


then

$$
z^{l}=\sum_{k \geq l} F_{k l} f_{k}(z) \quad \text { for all } l \in \mathbf{Z}^{s} .
$$

Obviously for any diagonal sequence $f$ there exists a uniquely determined inverse sequence $\mathfrak{F}$. In particular $F_{k k}=f_{k k}^{-1}$ is invertible in $\mathbf{A}$; therefore $\mathfrak{F}$ is also a diagonal sequence. Comparing the coefficients of $z^{n}$ in (3.3) leads to the relation

$$
\sum_{n \geq k \geq l} f_{n k} F_{k l}=\delta_{n l}
$$

Defining the operator $U_{\mathfrak{f}} z^{k}=f_{k}(z)$ by extending it linearly to $\overline{L s}(z)$, we see that $U_{\mathfrak{f}} F_{l}(z)=z^{l}$ by (3.3); moreover this implies $U_{\mathfrak{f}}^{-1}=U_{\mathfrak{F}}$. Applying $U_{\mathfrak{f}}^{-1}$ to (3.1) yields

$$
z^{k}=\sum_{n \geq k} f_{n k} F_{n}(z) .
$$

Again comparing the coefficients of $z^{m}$, we have

$$
\sum_{m \geq n \geq k} F_{m n} f_{n k}=\delta_{m k} .
$$

Identities (3.3)-(3.6) are equivalent. The "inverse relations" [22, Chapters 2, 3] are essentially based on identities (3.4) and (3.6), respectively. Concerning the connection between inverse relations and the Lagrange formula, see $[1,18,19$ and 12, Introduction]. In fact (3.4) and (3.6) simply say that the matrices $\left(f_{n k}\right)_{n, k \in \mathbf{Z}^{s}}$ and $\left(F_{k l}\right)_{k, l \in \mathbf{Z}^{*}}$ are inverses of each other. This point of view leads to the concept of "recursive matrices" introduced by Barnabei, Brini and Nicoletti (see [24] and the references cited there).

As indicated in the introduction, we need a sequence $\tilde{\mathfrak{f}}=\left(\tilde{f}_{k}(z)\right)_{k \in \mathbf{Z}^{s}}$ satisfying the orthogonality relation

$$
\left\langle f_{k}(z), \tilde{f}_{n}(z)\right\rangle=\delta_{n k}
$$

Using (3.6) we get

$$
\tilde{f}_{k}(z)=\sum_{l \leq k} F_{k l} z^{-l}
$$

Analogously, by (3.4),

$$
\tilde{F}_{n}(z)=\sum_{k \leq n} f_{n k} z^{-k}
$$

\section{The main theorems.}

THEOREM 1. Given $M_{1}$ and $M_{2}$ modules over the integral domain $\mathbf{A},($,$) a$ bilinear form from $M_{1} \times M_{2}$ into $\mathbf{A}$, and $I$ a set of indices, let $\left(f_{k}\right)_{k \in I}$ be a sequence of elements of $M_{1}$ with the property

(4.1) $\left(f_{k}, h\right)=0$ for all $k \in I$ if and only if $h=0$.

Let $U_{j}$ and $V$ denote linear operators on $M_{1}, V$ bijective, and let $g_{j}$ be arbitrary functions from $I$ into $\mathbf{A}$ such that

(4.2) $U_{j} f_{k}=g_{j}(k) V f_{k}$ for all $k \in I$ and $j=1,2, \ldots, r$. 
(4.3) For all indices $m, n \in I, m \neq n$, there exists a $j$ with $1 \leq j \leq r$ and $g_{j}(m) \neq g_{j}(n)$.

Suppose there exist the adjoint operators of $V, V^{-1}$ and $U_{j}$ denoted by $V^{*}, V^{-1^{*}}$ and $U_{j}^{*}\left(\right.$ this means $(V f, h)=\left(f, V^{*} h\right)$ for all $f \in M_{1}$ and $h \in M_{2}$, etc. $)$.

(A) If the system

$$
U_{j}^{*} h_{k}=g_{j}(k) V^{*} h_{k}, \quad k \in I, j=1,2, \ldots, r,
$$

has a nontrivial solution $\left(h_{k}\right)_{k \in I}-i$.e., $h_{k} \neq 0$ for all $k \in I$-then $\left(f_{k}, V^{*} h_{n}\right)=$ $\left(f_{k}, V^{*} h_{k}\right) \delta_{n k}$, where $\left(f_{k}, V^{*} h_{k}\right) \neq 0$ for all $k \in I$. If $\left(f_{k}, V^{*} h_{k}\right)$ is an invertible element of $\mathbf{A}$ for all $k \in I$, then there exists a (unique) sequence $\left(\tilde{f}_{k}\right)_{k \in I}$ of elements of $M_{2}$ satisfying

$$
\left(f_{k}, \tilde{f}_{n}\right)=\delta_{n k}, \quad n, k \in I
$$

and given by

$$
\tilde{f}_{k}=\left(f_{k}, V^{*} h_{k}\right)^{-1} \cdot V^{*} h_{k} .
$$

(B) Let $\left(f_{k}\right)_{k \in I}, U_{j}, V$ and $g_{j}$ be defined as above such that (4.1) and (4.2) hold. For a sequence $\left(\tilde{f}_{k}\right)_{k \in I}$ satisfying (4.5) the equations

$$
U_{j}^{*} V^{-1^{*}} \tilde{f}_{k}=g_{j}(k) \tilde{f}_{k}, \quad k \in I, j=1,2, \ldots, r,
$$

hold.

ProOF. (A) Suppose $\left(h_{k}\right)_{k \in I}$ satisfies (4.4). By using (4.2) we have

$$
\begin{aligned}
g_{j}(k)\left(f_{k}, V^{*} h_{n}\right) & =g_{j}(k)\left(V f_{k}, h_{n}\right)=\left(U_{j} f_{k}, h_{n}\right) \\
& =\left(f_{k}, U_{j}^{*} h_{n}\right)=g_{j}(n)\left(f_{k}, V^{*} h_{n}\right) .
\end{aligned}
$$

From (4.3) this immediately implies $\left(f_{k}, V^{*} h_{n}\right)=0$ for $n \neq k$. For $n=k$ suppose $\left(f_{n}, V^{*} h_{n}\right)=0$ for some $n \in I$. Together with the above calculation this means $\left(f_{k}, V^{*} h_{n}\right)=0$ for all $k \in I$. From (4.1) we conclude $V^{*} h_{n}=0$. It is easy to show that with our assumptions $V^{*}$ is bijective, and, therefore, $h_{n}=0$, in contradiction to the condition $h_{k} \neq 0$ for all $k \in I$.

Defining $\tilde{f}_{k}=\left(f_{k}, V^{*} h_{k}\right)^{-1} V^{*} h_{k}$, we evaluate

$$
\left(f_{k}, \tilde{f}_{n}\right)=\left(f_{n}, V^{*} h_{n}\right)^{-1}\left(f_{k}, V^{*} h_{n}\right)=\delta_{n k} .
$$

The uniqueness of the sequence $\left(\tilde{f}_{k}\right)_{k \in I}$ is obvious because of (4.1).

(B) Suppose $\left(f_{k}, \tilde{f}_{n}\right)=\delta_{n k}$ for all $n, k \in I$ by (4.2), we get

$$
\begin{aligned}
\left(f_{k}, U_{j}^{*} V^{-1^{*}} \tilde{f}_{n}\right) & =\left(V^{-1} U_{j} f_{k}, \tilde{f}_{n}\right)=g_{j}(k)\left(f_{k}, \tilde{f}_{n}\right) \\
& =g_{j}(n) \delta_{n k}=\left(f_{k}, g_{j}(n) \tilde{f}_{n}\right)
\end{aligned}
$$

which, combined with (4.1), proves (4.7).

We choose $M_{1}=M_{2}=\overline{L s}(z)$ and $I=\mathbf{Z}^{s}$. A usually can be considered a field. In this case $\left(f_{k}, V^{*} h_{k}\right)$ in Theorem 1(A) automatically is invertible because it is not zero. For the $q$-analogues we choose $\mathbf{A}=\mathbf{K}(q)$, the set of all rational functions in the indeterminates $q_{1}, q_{2}, \ldots, q_{s}$ over the field $\mathbf{K}$. The bilinear form we use is $\langle$,$\rangle ,$ as in $\S 2$. For the sequence $\left(f_{k}\right)_{k \in I}$ we choose a diagonal sequence of $\overline{L s}(z)$ (defined by (3.1)). Then condition (4.1) certainly holds. The Lagrange formulas then have the following form. 
COROLlARY 2. Let $\left(f_{k}(z)\right)_{k \in Z^{\text {s }}}$ be a diagonal sequence and $U_{j}, V$ linear operators as in Theorem 1 satisfying (4.1)-(4.3). If $g(z) \in \overline{L s}(z)$ and $\left(\tilde{f}_{k}(z)\right)_{k \in \mathbf{Z}^{s}}$ is given by (4.6), where the sequence $\left(h_{k}(z)\right)_{k \in \mathbf{Z}}$, with $h_{k}(z) \neq 0$ for all $k \in \mathbf{Z}^{s}$, satisfies (4.4), then the following hold: The coefficients in the expansion

$$
g(z)=\sum_{k \in \mathbf{Z}^{s}} c_{k} f_{k}(z)
$$

are computed by

$$
c_{n}=\left\langle g(z), \tilde{f}_{n}(z)\right\rangle
$$

or, if $\left\langle f_{n}, V^{*} h_{n}\right\rangle$ and $g_{j}(n)$ are invertible in $\mathbf{A}$,

$$
c_{n}=g_{j}(n)^{-1}\left\langle f_{n}, V^{*} h_{n}\right\rangle^{-1}\left\langle U_{j} g(z), h_{n}(z)\right\rangle .
$$

ProOF. The first formula is obvious because of (4.5). The second is just a rearrangement of the first, where we use (4.4) and (4.6).

The first version of Lagrange formula (1.1) corresponds to the second expression for $c_{n}$; the second version (1.2), to the first. When $s=1$ in the "ordinary" Lagrange formula, the form (4.9) (i.e., the first version) is simpler and usually easier to use than (4.8) (the second version). Because of the complexity of the operators $U_{j}$, in general (4.9) will be rather complicated or even inapplicable.

For a diagonal sequence $\left(f_{k}(z)\right)_{k \in \mathbf{Z}^{s}}$, the procedure of finding a Lagrange formula for this sequence is as follows: First we try to find a system of "eigenvalue" equations for the $f_{k}(z)$ 's of the form (4.2) where the operators satisfy the conditions of Theorem 1. Then we establish the dual system (4.4) deduced from the system above. Next we try to find a sequence $\left(h_{k}(z)\right)_{k \in \mathbf{Z}^{s}}$ of nontrivial solutions of the dual system and from this compute $\left(\tilde{f}_{k}(z)\right)_{k \in \mathbf{Z}^{s}}$ the system orthonormal to

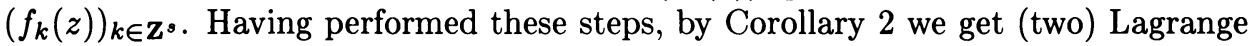
formulas for $\left(f_{k}(z)\right)_{k \in \mathbf{Z}^{s}}$. We demonstrate this method by deriving the classical Lagrange inversion formulas (1.1) and (1.2).

But first we need the adjoint operators relative to the bilinear form $\langle$,$\rangle which$ belong to the elementary operators introduced in $\S 2$. They are listed below. Given $a(z) \in \overline{L s}(z)$, the multiplication operator $a(z)$ is defined by $a(z)(b(z))=a(z) \cdot b(z)$. Then

$$
\begin{gathered}
a(z)^{*}=a(z), \quad\left(z_{i} D_{i}\right)^{*}=-z_{i} D_{i}, \quad \varepsilon_{i}^{\left(q_{i}\right)^{*}}=\varepsilon_{i}^{\left(1 / q_{i}\right)}, \\
\left(z_{i} D_{i}^{\left(q_{i}\right)}\right)^{*}=-\frac{1}{q_{i}} z_{i} D_{i}^{\left(1 / q_{i}\right)}=-\varepsilon_{i}^{\left(1 / q_{i}\right)} z_{i} D_{i}^{\left(q_{i}\right)} .
\end{gathered}
$$

These identities are easily verified. We prove the second. For all $k, n \in \mathbf{Z}^{s}$,

$$
\left\langle z_{i} D_{i} z^{k}, z^{n}\right\rangle=k_{i} \delta_{k,-n}=-n_{i} \delta_{k,-n}=\left\langle z^{k},-z_{i} D_{i} z^{n}\right\rangle .
$$

By linearity we can extend this to $a(z), b(z) \in \overline{L s}(z)$ :

$$
\left\langle z_{i} D_{i} a(z), b(z)\right\rangle=\left\langle a(z),-z_{i} D_{i} b(z)\right\rangle .
$$

Next we introduce another class of operators, the shift operators. Given a sequence $\mathfrak{f}=\left(f_{k}(z)\right)_{k \in \mathbf{Z} \boldsymbol{s}}$, we set

$$
{ }_{\mathfrak{f}} S_{i} f_{k}=f_{k-e_{i}}, \quad{ }_{\mathrm{f}} T_{i} f_{k}=f_{k+e_{i}} .
$$


If $\tilde{\mathfrak{f}}=\left(\tilde{f}_{k}(z)\right)_{k \in I}$ satisfies (3.7), then elementary considerations show that

$$
\begin{array}{ll}
{ }_{\mathfrak{f}} S_{i}^{*} \tilde{f}_{k}=\tilde{f}_{k+e_{i}}, & { }_{\mathfrak{f}} S_{i}^{*}={ }_{\mathfrak{f}} T_{i}, \\
{ }_{\mathfrak{f}} T_{i}^{*} \tilde{f}_{k}=\tilde{f}_{k-e_{i}}, & { }_{\mathfrak{f}} T_{i}^{*}={ }_{\mathfrak{f}} S_{i} .
\end{array}
$$

Moreover for $U_{\mathfrak{F}}$ holds:

$$
U_{\mathfrak{F}}^{*} z^{-k}=\tilde{f}_{k}(z)
$$

Now we turn to the one-variable Lagrange formula (see also [16]). We take $\mathbf{A}=\mathbf{K}$ a field of characteristic zero, $s=1$ and $f_{k}(z)=f^{k}(z)$ for a fixed formal power series $f(z)$ with $f(0)=0$ and $f^{\prime}(0) \neq 0$. This assures us of (3.1). Hence $f=\left(f_{k}(z)\right)_{k \in \mathbf{Z}}$ is a diagonal sequence. Thus

$$
z D f_{k}(z)=k \frac{z f^{\prime}(z)}{f(z)} f_{k}(z)
$$

Now applying Theorem $1(\mathrm{~A})$, for $r=1, U_{1}=z D, V=z f^{\prime}(z) / f(z), g_{1}(k)=k$, and (4.10) we get the dual equation

$$
-z D h_{k}(z)=k \frac{z f^{\prime}(z)}{f(z)} h_{k}(z)
$$

from which we immediately obtain $h_{k}(z)=f^{-k}(z)$. So

$$
\tilde{f}_{k}(z)=\frac{z f^{\prime}(z)}{f(z)} h_{k}(z)=\frac{z f^{\prime}(z)}{f^{k+1}(z)}
$$

by (4.6) since

$$
\left\langle f^{k}(z), \frac{z f^{\prime}(z)}{f^{k+1}(z)}\right\rangle=L_{0} \frac{z f^{\prime}(z)}{f(z)}=1 .
$$

Corollary 2 then reads as follows. For $g(z) \in \overline{L s}(z)$ the coefficients in the expansion $g(z)=\sum_{k \in \mathbf{Z}} c_{k} f^{k}(z)$ can be computed by

or

$$
c_{n}=\left\langle g(z), \frac{z f^{\prime}(z)}{f^{n+1}(z)}\right\rangle=\left\langle z^{0}\right\rangle g(z) \cdot \frac{z f^{\prime}(z)}{f^{n+1}(z)}
$$

$$
c_{n}=\frac{1}{n}\left\langle z D g(z), f^{-n}(z)\right\rangle=\left\langle z^{-1}\right\rangle \frac{1}{n} \frac{g^{\prime}(z)}{f^{n}(z)} \quad \text { for } n \neq 0 .
$$

These are exactly formulas (1.2) and (1.1).

In $\S 6$ it will be necessary to transfer eigenvalue equations of $\left(f_{k}(z)\right)_{k \in \mathbf{Z}}$ of the form (4.2) to the inverse sequence $\left(F_{k}(z)\right)_{k \in Z}$. We demonstrate this first for $f_{k}(z)=$ $f^{k}(z)$ before stating a general result. Suppose $F(f(z))=z$. The coefficients of the fps $F(z)$ are determined uniquely. We start with (4.13), which can be rewritten as

$$
z D f_{k}(z)=k \frac{F(f(z)) f^{\prime}(F(f(z)))}{f(z)} f_{k}(z) \text {. }
$$

Since, as is well known, $f^{\prime}(F(z))=1 / F^{\prime}(z)$, this is equivalent to

$$
z D f_{k}(z)=k \frac{F\left({ }_{\mathfrak{f}} T\right)}{{ }_{\mathfrak{f}} T F^{\prime}\left({ }_{\mathfrak{f}} T\right)} f_{k}(z) \text {. }
$$


The dual identity for $\left(\tilde{f}_{k}(z)\right)_{k \in \mathbf{Z}}$ is, according to Theorem 1(B) and using (4.10) and (4.11),

$$
-z D \frac{{ }_{\mathfrak{f}} S F^{\prime}\left({ }_{\mathrm{f}} S\right)}{F\left({ }_{\mathfrak{f}} S\right)} \tilde{f}_{k}(k)=k \tilde{f}_{k}(z) .
$$

Suppose $t F^{\prime}(t) / F(t)=\sum_{j=0}^{\infty} \Phi_{j} t^{j}$. Then comparing the coefficients of $z^{-l}$ by (3.8), leads to

$$
l \sum_{j=0}^{\infty} \Phi_{j} F_{k-j, l}=k F_{k l} .
$$

Multiplying both sides by $z^{k}$ and summing up over all $k \in \mathbf{Z}$ yield, with respect to (3.2),

$$
l \frac{z F^{\prime}(z)}{F(z)} F_{l}(z)=z D F_{l}(z),
$$

the eigenvalue equation for $F_{l}(z)=F^{l}(z)$.

The next theorem gives the general background of these considerations. But first we need some notation.

DEFINITION 3. The linear operator $\mathcal{L}(\mathfrak{f}, d, \mathcal{C})$ is called a generalized shift for the sequence $\mathfrak{f}=\left(f_{k}(z)\right)_{k \in \mathbf{Z}}$ of degree $d \in \mathbf{Z}^{s}$ and the associated sequence $C=\left(c_{k}\right)_{k \in \mathbf{Z}^{s}}$ with $c_{k} \in \mathbf{A}$ if

$$
\mathcal{L}(\mathfrak{f}, d, C) f_{k}(z)=c_{k} f_{k-d}(z) .
$$

All operators previously defined (with the exception of $U_{\mathfrak{f}}$ ) are generalized shifts (or sums of them):

$$
\begin{aligned}
z_{i}^{m} & =\mathcal{L}\left(\underline{1},-m e_{i},(1)_{k \in \mathbf{Z}^{s}}\right), \\
D_{i} & =\mathcal{L}\left(\underline{1}, e_{i},\left(k_{i}\right)_{k \in \mathbf{Z}^{s}}\right), \\
z_{i} D_{i} & =\mathcal{L}\left(\underline{1}, 0,\left(k_{i}\right)_{k \in \mathbf{Z}^{s}}\right), \\
\varepsilon_{i}^{\left(q_{i}\right)} & =\mathcal{L}\left(\underline{1}, 0,\left(q_{i}^{k_{i}}\right)_{k \in \mathbf{Z}^{s}}\right), \\
{ }_{\mathfrak{f}} S_{i} & =\mathcal{L}\left(\mathfrak{f}, e_{i},(1)_{k \in \mathbf{Z}^{s}}\right), \\
{ }_{\mathfrak{f}} T_{i} & =\mathcal{L}\left(\mathfrak{f},-e_{i},(1)_{k \in \mathbf{Z}^{s}}\right) .
\end{aligned}
$$

Note that the weak composition rule

$$
\mathcal{L}\left(\mathfrak{f}, d_{1}, \mathcal{C}\right) \mathcal{L}\left(\mathfrak{f}, d_{2}, \mathfrak{B}\right)=\mathcal{L}\left(\mathfrak{f}, d_{1}+d_{2},\left(c_{k-d_{2}} \cdot b_{k}\right)_{k \in \mathbf{Z}^{s}}\right)
$$

holds. Moreover, writing

$$
\mathcal{L}(\mathfrak{f}, d, C) f_{k}(z)=c_{k} S^{d} f_{k}(z),
$$

where ${ }_{f} S^{d}=\prod_{i=1}^{s} f_{i}^{d_{i}}\left(d=\left(d_{1}, \ldots, d_{s}\right)\right)$, by applying Theorem 1(B), gives

$$
\mathcal{L}(\mathfrak{f}, d, C)^{*}{ }_{\mathfrak{f}}^{d} \tilde{f}_{k}(z)=c_{k} \tilde{f}_{k}(z)
$$

or, after substituting $k+d$ for $k$,

$$
\mathcal{L}(\mathfrak{f}, d, C)^{*} \tilde{f}_{k}(z)=c_{k+d} \tilde{f}_{k+d}(z) .
$$

Therefore

$$
\mathcal{L}\left(\mathfrak{f}, d,\left(c_{k}\right)_{k \in \mathbf{Z}^{*}}\right)^{*}=\mathcal{L}\left(\tilde{\mathfrak{f}},-d,\left(c_{k+d}\right)_{k \in \mathbf{Z}^{*}}\right) .
$$


LEMMA 4. Let $\mathfrak{f}=\left(f_{k}(z)\right)_{k \in \mathbf{Z}^{*}}$ be a diagonal sequence with inverse sequence

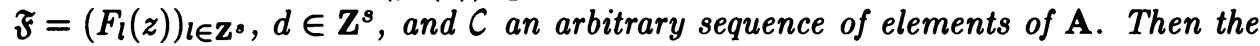
operator identities

$$
U_{\mathfrak{F}} \mathcal{L}(\mathfrak{f}, d, \mathcal{C})=\mathcal{L}(\underline{1}, d, C) U_{\mathfrak{F}}
$$

and

$$
U_{\mathfrak{F}} \mathcal{L}(\underline{1}, d, \mathcal{C})=\mathcal{L}(\mathfrak{F}, d, \mathcal{C}) U_{\mathfrak{F}}
$$

hold.

PROOF. This is easily verified by applying both sides of (4.19) to $f_{k}(z)$ and both sides of (4.20) to $z^{k}$, respectively.

For convenience we write ${ }^{*} \mathcal{L}(\mathfrak{f}, d, \mathcal{C})=\mathcal{L}(\underline{1}, d, \mathcal{C})$ and ${ }^{*} \mathcal{L}(\underline{1}, d, \mathcal{C})=\mathcal{L}(\mathfrak{F}, d, \mathcal{C})$.

THEOREM 5. Let $\mathfrak{f}=\left(f_{k}(z)\right)_{k \in Z}$ s be a diagonal sequence with inverse sequence $\mathfrak{F}=\left(F_{l}(z)\right)_{l \in \mathbf{Z}}$ and $L=\left\{L_{n} / n \in \mathbf{N}\right\}$ a set of generalized shifts for either $\mathfrak{f}$ or $\underline{1}$. $M_{i}(L)$ denote monomials of elements of $L$. Then

$$
\sum_{i} M_{i}(L)=0 \quad \text { if and only if } \sum_{i} M_{i}\left({ }^{*} L\right)=0,
$$

where $M_{i}\left({ }^{*} L\right)$ means the monomial obtained from $M_{i}(L)$ by replacing each $L_{n}$ by ${ }^{*} L_{n}$.

ProOF. By Lemma $4, U_{\mathfrak{F}} L_{n}={ }^{*} L_{n} U_{\mathfrak{F}}$. This implies $U_{\mathfrak{F}} M_{i}(L)=M_{i}\left({ }^{*} L\right) U_{\mathfrak{F}}$ and

$$
U_{\mathfrak{F}} \sum_{i} M_{i}(L)=\sum_{i} M_{i}\left({ }^{*} L\right) U_{\mathfrak{F}} .
$$

Since $U_{\mathfrak{F}}$ is bijective, the equivalence of the above operator identities is proved.

Let us try out this result by applying it to (4.15), which in this new terminology is written

$$
\mathcal{L}\left(\underline{1}, 0,(k)_{k \in \mathbf{Z}}\right)-\sum_{i=0}^{\infty} \Phi_{i}^{-1} \mathcal{L}\left(\mathfrak{f},-i,(k)_{k \in \mathbf{Z}}\right)=0,
$$

where $\sum_{i=0}^{\infty} \Phi_{i}^{-1} z^{i}=F(z) / z F^{\prime}(z)$, by definition. By Theorem 5 , which simply says that $f$ is to be replaced by $\underline{1}$ and $\underline{1}$ by $\mathcal{F}$, respectively, this becomes

$$
\mathcal{L}\left(\mathfrak{F}, 0,(k)_{k \in \mathbf{Z}}\right)-\sum_{i=0}^{\infty} \Phi_{i}^{-1} \mathcal{L}\left(\underline{1},-i,(k)_{k \in \mathbf{Z}}\right)=0 .
$$

Applying this identity to $F_{l}(z)$ gives

$$
k F_{l}(z)-\frac{F(z)}{z F^{\prime}(z)} z D F_{l}(z)=0
$$

and

as desired.

$$
z D F_{l}(z)=k \frac{z F^{\prime}(z)}{F(z)} F_{l}(z),
$$

From Theorem 1 and Definition 3 the eigenvalue equation (4.2) could also be written

$$
U_{j} f_{k}(z)=V \mathcal{L}\left(\mathfrak{f}, 0,\left(g_{j}(n)\right)_{n \in \mathbf{Z}^{s}}\right) f_{k}(z) .
$$


This equation is valid for all $f_{k}(z)$, which is supposed to be a diagonal sequence; hence

$$
U_{j}=V \mathcal{L}\left(\mathfrak{f}, 0,\left(g_{j}(n)\right)_{\left.n \in \mathbf{Z}^{*}\right)} .\right.
$$

Then the adjointed operators must also be equal.

$$
U_{j}^{*}=\mathcal{L}\left(\tilde{\mathfrak{f}}, 0,\left(g_{j}(n)\right)_{n \in \mathbf{Z}^{*}}\right) V^{*}
$$

by (4.18). This identity is applied to $V^{*-1} \tilde{f}_{k}(z)$ to get

$$
U_{j}^{*} V^{*-1} \tilde{f}_{k}(z)=g_{j}(k) \tilde{f}_{k}(z)
$$

which is (4.7).

5. The Lagrange-Good formula. In this section we start with $s$ fps $f_{i}(z)$ $(i=1,2, \ldots, s)$ in the variables $z_{1}, \ldots, z_{s}$ with coefficients in the integral domain $\mathbf{A}$ and the property $\left.f_{i}(z)\right|_{z_{i}=0}=0$ and $\left.\left(D_{i} f_{i}\right)(z)\right|_{z_{i}=0}$ is invertible in $\mathbf{A}$ for all $i$. Set $f(z)=\left(f_{1}(z), f_{2}(z), \ldots, f_{s}(z)\right)$. Then the sequence $f=\left(f^{k}(z)\right)_{k \in \mathbf{Z}^{s}}$, where $f^{k}(z)=f_{1}^{k_{1}}(z) f_{2}^{k_{2}}(z) \cdots f_{s}^{k_{s}}(z)$ is a diagonal sequence. Differentiation with respect to $z_{i}$ yields

$$
z_{i} D_{i} f^{k}(z)=\sum_{j=1}^{s} k_{j} \frac{z_{i}\left(D_{i} f_{j}\right)(z)}{f_{j}(z)} f^{k}(z), \quad i=1,2, \ldots, s .
$$

From these $s$ equations we are able to compute the $k_{i}$ 's by Cramer's rule, and thus we will get $s$ eigenvalue equations in the sense of Theorem 1 .

We write for convenience $[f](z)$ for the matrix

$$
\left(\frac{z_{i}\left(D_{i} f_{j}\right)(z)}{f_{j}(z)}\right)_{i, j=1}^{s}
$$

Its determinant is denoted by $\Delta f(z)$. Let $\Delta_{i j} f(z)$ be the determinant of the matrix obtained from $[f](z)$ by omitting the $i$ th row and $j$ th column. Then

$$
\left(\sum_{l=1}^{s}(-1)^{j+l} \Delta_{l j} f(z) z_{l} D_{l}\right) f^{k}(z)=k_{j} \Delta f(z) f^{k}(z), \quad j=1,2, \ldots, s .
$$

With $U_{j}=\sum_{l=1}^{s}(-1)^{j+l} \Delta_{l j} f(z) z_{l} D_{l}, V=\Delta f(z)$ and $g_{j}(k)=k_{j}$, we can apply Theorem 1 , since all other conditions are satisfied. First we compute $U_{j}^{*}$ :

$$
\begin{aligned}
U_{j}^{*} & =\left(\sum_{l=1}^{s}(-1)^{j+l} \Delta_{l j} f(z) z_{l} D_{l}\right)^{*} \\
& =-\sum_{l=1}^{s}(-1)^{j+l} z_{l} D_{l} \Delta_{l j} f(z) \\
& =-\sum_{l=1}^{s}(-1)^{j+l} \Delta_{l j} f(z) z_{l} D_{l}-\sum_{l=1}^{s}(-1)^{j+l} z_{l}\left(D_{l} \Delta_{l j} f\right)(z) .
\end{aligned}
$$

We shall show that the second sum in this expression is equal to zero. When this is done (4.4) reads

$$
-\left(\sum_{l=1}^{s}(-1)^{j+l} \Delta_{l j} f(z) z_{l} D_{l}\right) h_{k}(z)=k_{j} \Delta f(z) h_{k}(z) .
$$


By comparing this relation with (5.2), we immediately get the solutions

$$
h_{k}(z)=f^{-k}(z) \text {. }
$$

Since $\left\langle f_{k}(z), V^{*} h_{k}(z)\right\rangle=L_{0} \Delta f(z)=1$, by (4.6) we obtain

$$
\tilde{f}_{k}(z)=\Delta f(z) f^{-k}(z)=z^{e} \frac{\delta f(z) / \delta z}{f^{k+e}(z)}
$$

with $\delta f(z) / \delta z$ the Jacobian of $f$.

We now must prove $\sum_{l=1}^{s}(-1)^{j+l} z_{l}\left(D_{l} \Delta_{l j} f\right)(z)=0$. Let $\Sigma_{s}$ be the set of bijections on the set of integers between 1 and $s$. Then

$$
\begin{aligned}
\sum_{l=1}^{s}( & -1)^{j+l} z_{l}\left(D_{l} \Delta_{l j} f\right)(z) \\
= & \sum_{\pi \in \Sigma_{o}} \operatorname{sgn} \pi z_{\pi(j)} D_{\pi(j)}\left(\prod_{\substack{i=1 \\
i \neq j}} \frac{z_{\pi(i)}\left(D_{\pi(i)} f_{i}\right)(z)}{f_{i}(z)}\right) \\
= & \sum_{\pi \in \Sigma_{s}} \operatorname{sgn} \pi \sum_{\substack{k=1 \\
k \neq j}}^{s} \prod_{\substack{i=1 \\
i \neq j, k}} \frac{z_{\pi(i)}\left(D_{\pi(i)} f_{i}\right)(z)}{f_{i}(z)} \\
& \cdot\left(\frac{z_{\pi(j)} z_{\pi(k)}\left(D_{\pi(j)} D_{\pi(k)} f_{k}\right)(z)}{f_{k}(z)}-\frac{z_{\pi(j)}\left(D_{\pi(j)} f_{k}\right)(z) z_{\pi(k)}\left(D_{\pi(k)} f_{k}\right)(z)}{f_{k}^{2}(z)}\right) \\
= & \sum_{\pi \in \Sigma_{o}} \operatorname{sgn} \pi \sum_{\substack{k=1 \\
k \neq j}}^{s} A(\mathfrak{f}, j, k, \pi) \\
= & \sum_{\substack{k=1 \\
k \neq j}}^{s} \sum_{\pi \in \Sigma_{o}} \operatorname{sgn} \pi A(\mathfrak{f}, j, k, \pi) .
\end{aligned}
$$

We divide $\Sigma_{s}$ into two classes for fixed $j$ and $k: \Sigma_{s, k, j}^{(1)}=\{\sigma \mid \sigma(j)<\sigma(k)\}$ and $\Sigma_{s, k, j}^{(2)}$ the complementary set with respect to $\Sigma_{s}$; i.e., $\Sigma_{s, k, j}^{(2)}=\{\sigma \mid \sigma(j)>\sigma(k)\}$. We introduce the map $t_{j k}$ from $\Sigma_{s, k, j}^{(1)}$ onto $\Sigma_{s, k, j}^{(2)}$ by $t_{j k} \sigma=(\sigma(j) \sigma(k)) \sigma=\sigma(j k)$. This implies $\operatorname{sgn}\left(t_{j k} \sigma\right)=-\operatorname{sgn} \sigma$. Obviously $t_{j k}$ is a bijection for all $k \neq j$. Furthermore we note $A(\mathfrak{f}, j, k, \pi)=A\left(\mathfrak{f}, j, k, t_{j k} \pi\right)$. Hence

$$
\begin{aligned}
\sum_{\pi \in \Sigma_{。}} & \operatorname{sgn} \pi A(\mathfrak{f}, j, k, \pi) \\
& =\sum_{\pi \in \Sigma_{s, k, j}^{(1)}} \operatorname{sgn} \pi A(\mathfrak{f}, j, k, \pi)+\sum_{\pi \in \Sigma_{s, k, j}^{(2)}} \operatorname{sgn} \pi A(\mathfrak{f}, j, k, \pi) \\
& =\sum_{\pi \in \Sigma_{s, k, j}^{(1)}}\left(\operatorname{sgn} \pi A(\mathfrak{f}, j, k, \pi)+\operatorname{sgn} t_{j k} \pi A\left(\mathfrak{f}, j, k, t_{j k} \pi\right)\right) \\
& =0 .
\end{aligned}
$$

This is valid for each $k$; therefore (5.4) is equal to zero, as desired. 
Combined with Corollary 2, this completes the proof of

THEOREM 6 (LAGRANGE-GOOD FORMUla). The coefficients in the expansion

$$
g(z)=\sum_{k \in \mathbf{Z}^{s}} c_{k} f^{k}(z)
$$

are computed by

$$
c_{n}=\left\langle g(z), \frac{z^{e} \delta f(z) / \delta z}{f^{n+e}(z)}\right\rangle=\left\langle z^{-e}\right\rangle \frac{g(z) \delta f(z) / \delta z}{f^{n+e}(z)} .
$$

This is the analogue for (1.2).

Trying to get an analogue of (1.1) by (4.9) we get (if $n_{j}$ is invertible in $\mathbf{A}$ )

$$
c_{n}=n_{j}^{-1}\left\langle z^{0}\right\rangle\left[\sum_{l=1}^{s}(-1)^{j+l} \Delta_{l j} f(z) z_{l} D_{l} g(z)\right] f^{-n}(z)
$$

for $j=1,2, \ldots, s$. These $s$ formulas are put together in a symmetrical formula by multiplying the $j$ th formula by $n_{j} \cdot|n|^{-1}$ and then summing over all $j$. The result is

$$
c_{n}=|n|^{-1}\left\langle z^{0}\right\rangle\left[\sum_{l, j=1}^{s}(-1)^{j+l} \Delta_{l j} f(z) z_{l} D_{l} g(z)\right] f^{-n}(z)
$$

(if $|n|$ is invertible in $\mathbf{A}$ ), but it seems to be only of theoretical interest if $s>1$, since there is no improvement to formula (5.5) (which is the case for $s=1$ ), because in (5.7) $s^{2}$ determinants have to be computed, in (5.5) only one.

REMARK. (1) In the proofs of the Lagrange-Good formula the Jacobian of $f(z)$ is usually "pulled out of a hat"; generally (e.g., in $[\mathbf{1 6}, \mathbf{2 4}, \mathbf{2 7}]) L_{0} z^{e}(\delta f(z) / \delta z) f^{k}(z)$ $=\delta_{k e}$ is first proved. The advantage of our proof is that the Jacobian of $f(z)$, or better $\Delta f(z)$, appears naturally when transforming system (5.1) to system (5.2) in order to get eigenvalue equations which can be treated by Theorem 1 .

(2) Brini [24] and Henrici [27] extend the Lagrange-Good formula to a larger set of series. For a fixed $s \in \mathbf{N}$ consider fLs of the form

$$
a(z)=\sum_{l \in \mathbf{Z}^{s}} a_{l} z^{l},
$$

where for all $m \in \mathbf{Z}$ there is only a finite number of $a_{l}$ 's with $|l|<m$ which are different from zero. Let the set of all fLs of the form (5.8) be denoted by $\overline{\overline{L s}}(z)$. Then Brini and Henrici start with $f_{i}(z) \in \overline{\overline{L s}}(z)$ of the form

$$
f_{i}(z)=b_{i} z_{i}+\sum_{|l| \geq 2} b_{i l} z^{l}, \quad i=1,2, \ldots, s
$$

where the $b_{i}$ 's are invertible in A. They show that the Lagrange-Good formula remains true for these more general series. To establish this we can use our proof of Theorem 6 verbatim. The only difference is that when applying Theorem 1 and Corollary 2 we have to take $M_{1}=M_{2}=\overline{\overline{L s}}(z)$ instead of $\overline{L s}(z)$.

Concluding, we turn our attention to Abhyankar's [23] inversion formula, rediscovered independently by Garsia and Joni $[\mathbf{8}, \mathbf{9}, \mathbf{1 7}]$ and Viskov [28]. Let 
$f_{i}(z)(i=1,2, \ldots, s)$ be fls of the form (5.9) and $b_{i}=1, \mathfrak{f}=\left(f^{k}(z)\right)_{k \in \mathbf{Z}^{s}}$ and $\mathfrak{F}=\left(F^{l}(z)\right)_{l \in Z^{*}}$ its inverse sequence, where $F(z)=\left(F_{1}(z), \ldots, F_{s}(z)\right)$ with $F_{i}(z)$ being of the form (5.9), too. (Such a system $F(z)$ does exist; see [24, 27].) Abhyankar's formula gives an expression in terms of $f_{i}$ for the umbral operator $U_{\mathfrak{F}}$, i.e., the substitution by the inverse sequence $\mathfrak{F}$. The above-mentioned authors only prove it for $f_{i}$ being fps of the form (5.9). A slight modification of Henrici's [27] proof establishes its validity even for fLs of the form (5.9) with $b_{i}=1$.

THEOREM 7. Let $f_{i}(z), F_{i}(z)(i=1,2, \ldots, s)$ be defined as above. Then

$$
U_{\mathfrak{F}}=\sum_{m \geq 0} \frac{D^{m}}{m !} \frac{\delta f}{\delta z}(z) G^{m}(z)
$$

where $G_{i}(z)=z_{i}-f_{i}(z)$.

ProOF. Let $g(z)=\sum_{k \in \mathbf{Z}} c_{k} f^{k}(z)$. Then by definition of $U_{\mathfrak{F}}$,

$$
U_{\mathfrak{F}} g(z)=\sum_{k \in \mathbf{Z}^{s}} c_{k} z^{k}
$$

Therefore $c_{n}=\left\langle z^{n}\right\rangle U_{\mathfrak{F}} g(z)$. But considering Remark (2) above, we can compute $c_{n}$ by the Lagrange-Good formula (5.5). Hence

$$
\begin{aligned}
\left\langle z^{n}\right\rangle U_{\mathfrak{F}} g(z) & =\frac{\left\langle z^{0}\right\rangle z^{e} g(z) \delta f(z) / \delta z}{f^{n+e}(z)} \\
& =\frac{\left\langle z^{0}\right\rangle g(z)(\delta f(z) / \delta z) z^{-n}}{(1-G(z) / z)^{n+e}} \\
& =\left\langle z^{0}\right\rangle g(z) \frac{\delta f}{\delta z}(z) z^{-n} \sum_{m \geq 0}\left(\begin{array}{c}
n+m \\
m
\end{array}\right)(G(z) / z)^{m} \\
& =\sum_{m \geq 0}\left\langle z^{n+m}\right\rangle\left(\begin{array}{c}
n+m \\
m
\end{array}\right) \frac{\delta f}{\delta z}(z) G(z)^{m} g(z) \\
& =\left\langle z^{n}\right\rangle \sum_{m \geq 0} \frac{D^{m}}{m !} \frac{\delta f}{\delta z}(z) G(z)^{m} g(z) .
\end{aligned}
$$

Note that the application of the multinomial theorem was possible because the order (for definition see [27, (1.3)]) of $G_{i}(z) / z_{i}$ is at least 1 , since the $f_{i}$ 's are of the form (5.9) and $b_{i}=1$. Equation (5.11) holds for all $n \in \mathbf{Z}^{s}$; therefore

$$
U_{\mathfrak{F}} g(z)=\sum_{m \geq 0} \frac{D^{m}}{m !} \frac{\delta f}{\delta z}(z) G^{m}(z) g(z)
$$

which is true for all $g(z) \in \overline{\overline{L s}}(z)$. This furnishes the operator identity (5.10).

The generalization of Theorem 6 to $\overline{\overline{L s}}(z)$ seems to be a little artificial, but there are beautiful applications which can be found in $[\mathbf{2 7}, \S 5]$. For surveys and references concerning multivariable Lagrange formulas, see [27 and 26, Part II].

6. Garsia's $q$-analogue of the Lagrange formula. Here we take $s=1$ and $\mathbf{A}=\mathbf{K}(q)$ to be the set of all rational functions over a field $\mathbf{K}$ in the indeterminate $q$. Let $f(z)$ be an fps (in one variable $z$ ) over $\mathbf{K}(q)$ with $f(0)=0$ and $f^{\prime}(0) \neq 0$. 
The powers $f^{k}(z)$ are replaced by the " $q$-powers" $f(z) \cdot f(q z) \cdots f\left(q^{k-1} z\right)$ for $k \in \mathbf{N}$. To apply our method, we need an extension for $k \in \mathbf{Z}$. Hence we define

$$
f^{[k, q]}(z)= \begin{cases}f(z) \cdot f(q z) \cdots f\left(q^{k-1} z\right) & \text { for } k>0 \\ 1 & \text { for } k=0 \\ \frac{1}{f(z / q) f\left(z / q^{2}\right) \cdots f\left(z / q^{-k}\right)} & \text { for } k<0 .\end{cases}
$$

With the help of Garsia's [7] starring operator this could be written in closed form:

$$
f^{[k, q]}(z)=f^{*}(z) / f^{*}\left(q^{k} z\right) \quad \text { for } k \in \mathbf{Z} .
$$

This definition warrants the following properties for $k, l \in \mathbf{Z}$ :

$$
\begin{gathered}
f^{[k, q]}(z) \cdot f^{[l, q]}\left(q^{k} z\right)=f^{[k+l, q]}(z), \\
f^{[k, q]}(z) / f^{[l, q]}(z)=f^{[k-l, q]}\left(q^{l} z\right), \\
1 / f^{[k, q]}(z)=f^{[-k, 1 / q]}(z / q), \\
f^{[k, q]}(z)=f^{[k, 1 / q]}\left(q^{k-1} z\right) .
\end{gathered}
$$

These identities are easily verified by trying all cases.

Let $f_{k}(z)=f^{[k, q]}(z)$. Then $\mathfrak{f}=\left(f_{k}(z)\right)_{k \in \mathbf{Z}}$ is a diagonal sequence. Then

$$
f^{[1, q]}(z) f^{[k, q]}(q z)=f^{[k+1, q]}(z)
$$

by (6.1). But this is

$$
f(z) \varepsilon^{(q)} f_{k}(z)={ }_{\mathfrak{f}} T f_{k}(z) .
$$

By Theorem 1(B) the dual equation would be, by (4.10) and (4.11),

$$
\varepsilon^{(1 / q)} f(z)_{\tilde{\mathfrak{f}}} T \tilde{f}_{k}(z)=\tilde{f}_{k}(z) .
$$

This leads to

$$
\tilde{f}_{k+1}(z)=\tilde{f}_{k}(q z) / f(z),
$$

and therefore we get

$$
\tilde{f}_{k}(z)=\tilde{f}_{0}\left(q^{k} z\right) / f^{[k, q]}(z),
$$

which is proved inductively. The orthogonality relation (3.7) then becomes

$$
L_{0} f^{[k, q]}(z) \frac{\tilde{f}_{0}\left(q^{n} z\right)}{f^{[n, q]}(z)}=\delta_{n k} .
$$

Using (6.2) and replacing $z$ by $q^{-n} z$ give

$$
L_{0} f^{[k-n, q]}(z) \tilde{f}_{0}(z)=\delta_{n k},
$$

or, using (6.3),

$$
L_{0} \frac{\tilde{f}_{0}(z)}{f^{[n-k, 1 / q]}(z / q)}=\delta_{n k} .
$$

Setting $n-k=m$, we get

$$
L_{0} \frac{\tilde{f}_{0}(z)}{f^{[m, 1 / q]}(z / q)}=\delta_{m 0} .
$$


Since $\tilde{f}_{0}(z)$ is an fps, the last identity does not give any information about $\tilde{f}_{0}(z)$ when $m<0$. For $m \geq 0$ we have

$$
\left\langle z^{0}\right\rangle \frac{\tilde{f}_{0}(z)}{f(z / q) \cdots f\left(z / q^{m}\right)}=\delta_{m 0} .
$$

By this identity the coefficients of $\tilde{f}_{0}(z)$ can be successively evaluated. By comparing with Garsia [7, identity (1.7)], we see that our $\tilde{f}_{0}(z)$ is, in Garsia's notation, $z f^{0}(z) / f(z)$ or, vice versa,

$$
f^{0}(z)=(f(z) / z) \tilde{f}_{0}(z) .
$$

(In our context this is to be regarded as a definition. Moreover we remark that in Garsia's paper the roles of $f(z)$ and $F(z)$ are exchanged.)

With his roofing and starring operations, Garsia is able to give an explicit expression for his $f^{0}(z)$. This cannot be deduced by our method (starring is a nonlinear operator). We refer the reader to Garsia's paper. We intend to give a survey of the usefulness of our method by deriving and extending Garsia's results that do not involve roofing or starring operations within our setting, finding some new ones ((6.14), (6.24), Theorems 8 and 10), and proving Gessel's [10] $q$-Lagrange theorem.

Next we study properties of the inverse sequence $\mathfrak{F}=\left(F_{k}(z)\right)_{k \in \mathbf{Z}}$. Equation (3.3) in terms of the $q$-powers $f_{k}(z)=f^{[k, q]}(z)$ becomes, for $l=1$,

$$
\sum_{i=1}^{\infty} F_{i 1} f^{[i, q]}(z)=z .
$$

We denote the fps $F_{1}(z)=\sum_{i=1}^{\infty} F_{i 1} z^{i}$ by $F(z)$. Substituting $z$ for $q^{k} z$ and multiplying by $f^{[k, q]}(z)$ transform (6.9) into

$$
\sum_{i=1}^{\infty} F_{i 1} f^{[k+i, q]}(z)=q^{k} z f^{[k, q]}(z)
$$

with respect to (6.1). In the operator terminology of $\S 4$ this is

$$
\sum_{i=1}^{\infty} \mathcal{L}\left(\mathfrak{f},-i,\left(F_{j 1}\right)_{j \in \mathbf{Z}}\right)=\mathcal{L}\left(\underline{1},-1,(1)_{j \in \mathbf{Z}}\right) \mathcal{L}\left(\mathfrak{f}, 0,\left(q^{j}\right)_{j \in \mathbf{Z}}\right) .
$$

The dual equation, according to Theorem 5 , is

$$
\sum_{i=1}^{\infty} \mathcal{L}\left(\underline{\mathbf{1}},-i,\left(F_{j 1}\right)_{j \in \mathbf{Z}}\right)=\mathcal{L}\left(\mathfrak{F},-1,(1)_{j \in \mathbf{Z}}\right) \mathcal{L}\left(\underline{1}, 0,\left(q^{j}\right)_{j \in \mathbf{Z}}\right)
$$

or, in terms of the elementary operators,

$$
F(z)=\mathfrak{F}^{T \varepsilon^{(q)}} \text {. }
$$

Applying this operator identity to $\varepsilon^{(1 / q)} F_{l}(z)$ yields

$$
F(z) F_{l}(z / q)=F_{l+1}(z) .
$$

Combining this with $F_{1}(z)=F(z)$, we get immediately the surprising beautiful form

$$
F_{l}(z)=F^{[l, 1 / q]}(z)
$$


From this point of view Garsia calls $F(z)$ the right inverse of $f(z)$ (and $f(z)$ the left inverse of $F(z)$ ).

As a corollary we get

$$
\sum_{k} a_{k} z^{k}=\sum_{k} b_{k} f^{[k, q]}(z)
$$

if and only if

$$
\sum_{l} a_{l} F^{[l, 1 / q]}(z)=\sum_{l} b_{l} z^{l}
$$

The second equation is derived from the first by applying the operator $U_{f}^{-1}$, and vice versa.

By (6.5) the Lagrange formula (4.8) reads as follows: The coefficients in the expansion

$$
g(z)=\sum_{k \in \mathbf{Z}} c_{k} f^{[k, q]}(z)
$$

where $g(z) \in \overline{L s}(z)$, are given by

$$
c_{n}=\left\langle g(z), \frac{\tilde{f}_{0}\left(q^{n} z\right)}{f^{[n, q]}(z)}\right\rangle=\left\langle z^{0}\right\rangle g(z) \frac{\tilde{f}_{0}\left(q^{n} z\right)}{f^{[n, q]}(z)} .
$$

(By comparing with (1.2) we note that $\tilde{f}_{0}(z)$ is the $q$-analogue for $z f^{\prime}(z) / f(z)$; hence $f^{0}(z)$ by $(6.8)$ for $f^{\prime}(z)$.)

Interesting new facts arise by studying the adjoint of $U_{\mathfrak{F}}$. First we find an expression for the coefficients of $\tilde{f}_{0}(z) f^{[n, q]}(z)$. For $g(z)=z^{-k}$ the Lagrange formula yields for the expansion (6.12)

$$
c_{n}=L_{0}\left(z^{-k} \frac{\tilde{f}_{0}\left(q^{n} z\right)}{f^{[n, q]}(z)}\right) .
$$

On the other hand, by applying (6.11) to (6.12) we get

$$
c_{n}=\left\langle z^{n}\right\rangle F^{[-k, 1 / q]}(z)=L_{0}\left(z^{-n} F^{[-k, 1 / q]}(z)\right) .
$$

Therefore by changing $n$ into $-n$, we get

$$
L_{0}\left(z^{n} F^{[-k, 1 / q]}(z)\right)=L_{0}\left(z^{-k} \frac{\tilde{f}_{0}\left(q^{-n} z\right)}{f^{[-n, q]}(z)}\right) .
$$

On the left side we substitute $q^{-k} z$ for $z$; on the right, $q^{n} z$ for $z$. This is allowed because $L_{0}(a(z))=L_{0}(a(p z))$ for all $a(z) \in \overline{L s}(z)$ and constant $p$. Together with (6.2) this leads to

$$
L_{0}\left(z^{n} / F^{[k, 1 / q]}(z)\right)=L_{0}\left(z^{-k} \tilde{f}_{0}(z) f^{[n, q]}(z)\right)
$$

or

$$
\left\langle z^{k}\right\rangle \tilde{f}_{0}(z) f^{[n, q]}(z)=\left\langle z^{-n}\right\rangle 1 / F^{[k, 1 / q]}(z) .
$$

This identity plays a significant role in the proof of Gessel's theorem [10, Theorem 6.9]. In addition, it is easy to extend Garsia's $q$-Lagrange inversion formulas [7, 
identity (1.10) and 25, identity (5.6)] to integral $n$ and arbitrary $\Phi(z) \in \overline{L s}(z)$ with the help of (6.14). In fact each of them valid for all $n \in \mathbf{Z}$ and $\Phi(z) \in \overline{L s}(z)$ is equivalent to (6.14).

In particular, we get, for $n=0$,

$$
\left\langle z^{k}\right\rangle \tilde{f}_{0}(z)=\left\langle z^{0}\right\rangle \frac{1}{F^{[k, 1 / q]}(z)} .
$$

After we substitute $q^{k-1} z$ instead of $z$ on the right side, (6.4) implies

$$
\left\langle z^{k}\right\rangle \tilde{f}_{0}(z)=\left\langle z^{0}\right\rangle \frac{1}{F^{[k, q]}(z)} \quad\left(=\left\langle z^{0}\right\rangle F^{[-k, q]}(z)\right) .
$$

This briefly means, if we remember $(6.11)$, that $\tilde{f}_{0}(z)$ is the same for the left and right inverses of $F(z)$. (6.14) for $n=1$ and $k+1$ instead of $k$ is

$$
\left\langle z^{k}\right\rangle \frac{f(z)}{z} \tilde{f}_{0}(z)=\left\langle z^{-1}\right\rangle \frac{1}{F^{[k+1,1 / q]}(z)},
$$

which turns out to be Garsia's coefficient representation of his $f^{0}(z)[\mathbf{7}$, Theorem 1.3 ; compare to (6.8).

Now we consider $U_{\mathfrak{F}}^{*}$.

$$
\begin{aligned}
\left\langle z^{k}, U_{\mathfrak{F}}^{*} \frac{1}{F^{[-n, 1 / q]}(z)}\right\rangle & =\left\langle U_{\mathfrak{F}} z^{k}, \frac{1}{F^{[-n, 1 / q]}(z)}\right\rangle \\
& =\left\langle F^{[k, 1 / q]}(z), \frac{1}{F^{[-n, 1 / q]}(z)}\right\rangle \\
& =L_{0} F^{[k+n, 1 / q]}\left(q^{n} z\right)
\end{aligned}
$$

by (6.2). Using (6.3), we rewrite (6.14), for $n=0$, as

$$
\left\langle z^{k}\right\rangle \tilde{f}_{0}(z)=\left\langle z^{0}\right\rangle F^{[-k, 1 / q]}(q z)=L_{0} F^{[-k, 1 / q]}(p z)
$$

for an arbitrary constant $p$. Therefore

$$
\left\langle z^{k}, U_{\mathfrak{F}}^{*} \frac{1}{F^{[-n, 1 / q]}(z)}\right\rangle=\left\langle z^{-k-n}\right\rangle \tilde{f}_{0}(z)=\left\langle z^{k}, \tilde{f}_{0}(z) z^{n}\right\rangle .
$$

As valid for all $k \in \mathbf{Z}$

$$
U_{\mathfrak{F}}^{*} \frac{1}{F^{[-n, 1 / q]}(z)}=\tilde{f}_{0}(z) z^{n} .
$$

After using (6.3) again, we get

$$
\tilde{f}_{0}(z)^{-1} U_{\mathfrak{F}}^{*} \varepsilon^{(q)} F^{[n, q]}(z)=z^{n} .
$$

But according to (4.12) and (6.5),

$$
\begin{aligned}
\tilde{f}_{0}(z)^{-1} U_{\mathfrak{F}}^{*} \varepsilon^{(q)} z^{n} & =q^{n} \tilde{f}_{0}(z)^{-1} \tilde{f}_{-n}(z) \\
& =q^{n} \frac{\tilde{f}_{0}\left(q^{-n} z\right)}{\tilde{f}_{0}(z)} \frac{1}{f^{[-n, q]}(z)} \\
& =\left(q \frac{\tilde{f}_{0}(z / q)}{\tilde{f}_{0}(z)} f\left(\frac{z}{q}\right)\right)^{[n, 1 / q]}
\end{aligned}
$$


Therefore the right inverse of $F(z)$ is $q\left(\tilde{f}_{0}(z / q) / \tilde{f}_{0}(z)\right) f(z / q)$. This proves

THEOREM 8. Let $f(z)$ be the left inverse and ${ }_{1} f(z)$ the right inverse of $F(z)$. The coefficient of $z^{n}$ of the fps $\tilde{f}_{0}(z)$ is defined by (6.15). Then the relation

$$
{ }_{1} f(z)=q\left(\tilde{f}_{0}(z / q) / \tilde{f}_{0}(z)\right) \cdot f(z / q)
$$

holds, and the operator identity

$$
U_{\mathfrak{F}}^{*}=\tilde{f}_{0}(z) U_{1} \varepsilon^{(1 / q)}
$$

where ${ }_{1} f=\left({ }_{1} f^{[k, 1 / q]}(z)\right)_{k \in \mathbf{Z}}$.

Quite analogously read the results for $F(z)$, namely

$$
{ }_{1} F(z)=\frac{1}{q} \frac{\tilde{F}_{0}(q z)}{\tilde{F}_{0}(z)} F(q z)
$$

if ${ }_{1} F(z)$ denotes the left inverse of $f(z)$ and

$$
U_{\mathfrak{f}}^{*}=\tilde{F}_{0}(z) U_{1 \mathfrak{F}} \varepsilon^{(q)} .
$$

Because $U_{\mathfrak{F}}=U_{\mathfrak{f}}^{-1}$, we have by (6.20) and (6.22) that

$$
\tilde{f}_{0}(z) U_{1 \mathfrak{f}} \varepsilon^{(1 / q)}=\varepsilon^{(1 / q)} U_{1 \mathfrak{F}}^{-1} \tilde{F}_{0}(z)^{-1} .
$$

Combining (6.8) and (6.19) yields

$$
{ }_{1} f(z) \tilde{f}_{0}(z) / z=f^{0}(z / q)
$$

and, analogously,

$$
{ }_{1} F(z) \tilde{F}_{0}(z) / z={ }^{0} F(q z) .
$$

(Garsia has to distinguish between $f^{0}(z)$ and ${ }^{0} f(z)$ or $F^{0}(z)$ and ${ }^{0} F(z)$, respectively, because these fps are not independent of whether we take the right or left inverse. The advantage of our $\tilde{f}_{0}(z)$ is that we need not bother about this. For clarity $f^{0}(z)$ arises by taking the right inverse of $f(z) ;{ }^{0} f(z)$, by taking the left inverse of $f(z)$ or, in other words, the coefficient of $z^{k}$ in $f^{0}(z)$ is given by the right side of (6.16), and the coefficient of $z^{k}$ in ${ }^{0} f(z)$ is given by the right side of (6.16) after $q$ is replaced by $1 / q$ and $F$ is replaced by ${ }_{1} F$. The same holds analogously for $F^{0}(z)$ and ${ }^{0} F(z)$.)

Moreover, we state the operator identities

$$
U_{1 f 1}^{-1} f(z) \varepsilon^{(1 / q)}=z U_{1 f}^{-1}
$$

and

$$
U^{-1}{ }_{1} \mathfrak{F}_{1} F(z) \varepsilon^{(q)}=z U_{1}^{-1},
$$

which are verified by applying ${ }_{1} f^{[n, 1 / q]}(z)$ and ${ }_{1} F^{[n, q]}(z)$, respectively. Then (6.23) reduces to

$$
f^{0}(z) U_{1 \mathfrak{f}}=U_{1 \mathfrak{F}}^{-10} F(z)^{-1}
$$


Garsia's $q$-analogues of the chain rule for derivatives are special cases of this operator identity. Here it is convenient to adopt Garsia's notation for his $q$-analogue of functional composition:

$$
\Phi(\bar{f})=\sum_{k} \Phi_{k} f^{[k, q]}(z) \quad \text { or } \quad \Phi(\underline{F})=\sum_{l} \Phi_{l} F^{[l, 1 / q]}(z),
$$

respectively, with $\Phi(z)=\sum_{k} \Phi_{k} z^{k}$ an element of $\overline{L s}(z)$. Applying $(6.24)$ to ${ }^{0} F(z)$ then yields

$$
f^{0}(z)^{0} F(\underline{1} f)=1
$$

[7, Theorem 2.6]. By (6.19) and (6.8) we know that

$$
{ }_{1} f^{[k, 1 / q]}(z)=q^{k} \frac{\tilde{f}_{0}\left(z / q^{k}\right)}{f^{0}(z)} f^{[k+1,1 / q]}(z) \cdot \frac{1}{z},
$$

which transforms the last identity into

$$
\sum_{k=0}^{\infty}{ }^{0} F_{k} q^{k} f^{[k+1,1 / q]}(z) \tilde{f}_{0}\left(z / q^{k}\right)=z
$$

([7, Theorem 1.4], where $q$ is replaced by $1 / q$. The equality of $\tilde{f}_{0}(z)$ and Garsia's $e_{q}(z)$ are proved in Theorem 8). Applying (6.24) to 1 yields

$$
f^{0}(z)={ }^{0} F^{-1}(\underline{f})
$$

which is another $q$-analogue of $f^{\prime}(z)=F^{\prime}(f(z))^{-1}$.

Now we are in a position to give a new proof of Gessel's (strong) theorem [10, Theorem 6.9], which in our notation reads as follows.

THEOREM 9 (GESSEL). Let $f(z)$ satisfy

$$
f(z)=q z \sum_{n=0}^{\infty} g_{n} f^{[n, q]}(z)
$$

with $g(z)=\sum_{n=0}^{\infty} g_{n} z^{n}$ an fps with $g(0) \neq 0$. Let $\bar{f}(z)$ satisfy

$$
\bar{f}(z)=\frac{z}{q} \sum_{n=0}^{\infty} g_{n} \bar{f}^{[n, 1 / q]}(z) .
$$

Then there for $n, k \in \mathbf{Z}$,

$$
\left\langle z^{n}\right\rangle \tilde{f}_{0}(z) f^{[k, q]}(z)=q^{\left(\begin{array}{c}
n+1 \\
2
\end{array}\right)}\left\langle z^{n-k}\right\rangle g^{[n, 1 / q]}(z / q)
$$

holds, where $\tilde{f}_{0}(z)=(1-d(z))^{-1}$ with

$$
d(z)=z \sum_{i, j=0}^{\infty} g_{i+j+1} f^{[i, q]}(z) \bar{f}^{[j, 1 / q]}(z) .
$$

ProOF. Division by $z f(z)$ and substitution of $z / q$ instead of $z$ turn (6.28) into

$$
\frac{1}{z}=\sum_{n=-1}^{\infty} g_{n+1} f^{[n, q]}(z) .
$$


But $\sum_{n=-1}^{\infty} g_{n+1} z^{n}=g(z) / z$. If we set $F(z)=z / g(z)$, then $F^{[-1,1 / q]}(z / q)=$ $g(z) / z$. By (6.11) we obtain that the left inverse of $F(z / q)$ exactly satisfies (6.32). Therefore (because of uniqueness) $f(z)$ must be the left inverse of $F(z / q)$. Since $U_{\mathfrak{F}} \varepsilon^{(q)}[F(z / q)]^{[l, 1 / q]}=z^{l}$, we get $f(z)=U_{\mathfrak{F}} \varepsilon^{(q)} z=q\left(U_{\mathfrak{F}} z\right) . U_{\mathfrak{F}} z$ is the left inverse of $F(z)$; therefore $f(z) / q$ is the left inverse of $F(z)$. Similar considerations starting with (6.29) show that $q \bar{f}(z)$ is the right inverse of $F(z)$. This and (6.19) give

$$
\bar{f}(z)=q^{-1} \frac{\tilde{f}_{0}(z / q)}{\tilde{f}_{0}(z)} f(z / q)
$$

where $\tilde{f}_{0}(z)$ is defined as in Theorem 8 .

Since (6.30) is obviously just a rearrangement of (6.14) with $n$ and $k$ exchanged, only $1-1 / \tilde{f}_{0}(z)=d(z)$ is left to be proved. We have

$$
\begin{aligned}
& 1-\frac{1}{\tilde{f}_{0}(z)}= \frac{1}{\tilde{f}_{0}(z)}\left(\tilde{f}_{0}(z)-1\right) \\
&=\frac{1}{\tilde{f}_{0}(z)}\left(1-\varepsilon^{(1 / q)}\right)^{-1}\left(\tilde{f}_{0}(z)-\tilde{f}_{0}\left(\frac{z}{q}\right)\right) \\
&=\frac{1}{\tilde{f}_{0}(z)}\left(1-\varepsilon^{(1 / q)}\right)^{-1}\left(z \tilde{f}_{0}(z) \sum_{n=-1}^{\infty} g_{n+1} f^{[n, q]}(z)\right. \\
&\left.\quad-\left(\frac{z}{q}\right) \tilde{f}_{0}\left(\frac{z}{q}\right) \sum_{n=-1}^{\infty} g_{n+1} \bar{f}^{[n, 1 / q]}\left(\frac{z}{q}\right)\right)
\end{aligned}
$$

by (6.28) and (6.29). Using (6.33) and (6.4), we obtain

$$
\begin{aligned}
& 1-\frac{1}{\tilde{f}_{0}(z)}=\frac{1}{\tilde{f}_{0}(z)} \frac{1}{1-\varepsilon^{(1 / q)}}\left(\sum _ { n = 0 } ^ { \infty } g _ { n + 1 } \left(z \tilde{f}_{0}(z) f^{[n, q]}(z)\right.\right. \\
& \left.\left.\quad-\frac{z}{q^{n+1}} \tilde{f}_{0}\left(\frac{z}{q^{n+1}}\right) f^{[n, q]}\left(\frac{z}{q^{n+1}}\right)\right)\right) \\
& =\frac{1}{\tilde{f}_{0}(z)} \sum_{n=0}^{\infty} g_{n+1} \frac{1-\varepsilon^{(1 / q)^{n+1}}}{1-\varepsilon^{(1 / q)}}\left(z \tilde{f}_{0}(z) f^{[n, q]}(z)\right) \\
& =\frac{1}{\tilde{f}_{0}(z)} \sum_{n=0}^{\infty} g_{n+1} \sum_{j=0}^{n} \varepsilon^{(1 / q)^{j}}\left(z \tilde{f}_{0}(z) f^{[n, q]}(z)\right) \\
& =z \sum_{i, j=0}^{\infty} g_{i+j+1} q^{-j} \frac{\tilde{f}_{0}\left(q^{-j} z\right)}{\tilde{f}_{0}(z)} f^{[j, 1 / q]}\left(\frac{z}{q}\right) f^{[i, q]}(z) .
\end{aligned}
$$

Again using (6.33), we get the desired equation

$$
1-\frac{1}{\tilde{f}_{0}(z)}=z \sum_{i, j=0}^{\infty} g_{i+j+1} f^{[i, q]}(z) f^{[j, 1 / q]}(z) .
$$

The next theorem adds another identity of this rather strange type; it is a $q$ analogue of the chain rule for derivatives. 
THEOREM 10. Let $F(z)=\sum_{n=1}^{\infty} F_{n} z^{n}, f(z)$ be the left inverse and ${ }_{1} f(z)$ the right inverse of $F(z)$ and $f^{0}(z)$ as in (6.8). Then

$$
\sum_{i, j=0}^{\infty} F_{i+j+1} f^{[i, q]}(q z)_{1} f^{[j, 1 / q]}(z) f^{0}(z)=1 .
$$

Proof. By (6.19) and (6.8),

$$
\begin{aligned}
(1- & \left.\varepsilon^{(1 / q)}\right) \sum_{i, j=0}^{\infty} F_{i+j+1} f^{[i, q]}(q z){ }_{1} f^{[j, 1 / q]}(z) f^{0}(z) \\
& =\left(1-\varepsilon^{(1 / q)}\right) \sum_{i, j=0}^{\infty} F_{i+j+1} f^{[i, q]}(q z) q^{j} \frac{\tilde{f}_{0}\left(z / q^{j}\right)}{\tilde{f}_{0}(z)} f^{[j, 1 / q]}\left(\frac{z}{q}\right) \frac{\tilde{f}_{0}(z) f(z)}{z} \\
& =\left(1-\varepsilon^{(1 / q)}\right) \sum_{n=0}^{\infty} F_{n+1} \sum_{j=0}^{n} \varepsilon^{(1 / q)^{j}} \frac{\tilde{f}_{0}(z)}{z} f^{[n+1, q]}(z) \\
& =\sum_{n=0}^{\infty} F_{n+1}\left(1-\varepsilon^{(1 / q)^{n+1}}\right) \frac{\tilde{f}_{0}(z)}{z} f^{[n+1, q]}(z) \\
& =\frac{\tilde{f}_{0}(z)}{z} \sum_{n=0}^{\infty} F_{n+1} f^{[n+1, q]}(z)-\frac{\tilde{f}_{0}(z)}{z} \sum_{n=0}^{\infty} F_{n+1} f^{[n+1,1 / q]}(z) \\
& =\frac{\tilde{f}_{0}(z)}{z} z-\frac{\tilde{f}_{0}(z)}{z} z=0 .
\end{aligned}
$$

Therefore the left-hand side of (6.34) is equal to a constant, which is easily evaluated by setting $z=0$.

Obviously more identities of this type can be obtained in a similar manner. Indeed, a beautiful theory can be developed. But unfortunately it seems that only in Carlitz's special case, that is, $f(z)=z /(1-z)$, can the fps $f^{0}(z)$ and $\tilde{f}_{0}(z)$ be evaluted concretely. (There

$$
\left.f^{0}(z)=\frac{1}{(1-z)(1-z / q)} \quad \text { and } \quad \tilde{f}_{0}(z)=\frac{1}{1-z / q} .\right)
$$

A slight generalization of this example can be obtained from the same considerations Gessel makes in order to expand his $q$-Lagrange inversion formula of [11] to the inversion formula of [12]. (Riordan does the same in the opposite direction, when he derives the inverse relations of Legendre type by those of the Chebyshev type in $[22, \S 2.5]$.) We only briefly state the result of this procedure, making use of the terminology of $[\mathbf{1 8}]$, namely

$$
p_{\alpha}^{(q)}(1, z)=\frac{e_{q}\left(q^{\alpha} z /(1-q)\right)}{e_{q}(z /(1-q))}=\sum_{k=0}^{\infty}(-1)^{k} q^{\left(\begin{array}{c}
k \\
2
\end{array}\right)}\left[\begin{array}{l}
\alpha \\
k
\end{array}\right] z^{k}
$$

which is a $q$-analogue for $(1-z)^{\alpha}$. Let $d$ be a natural number. Then for $f(z)=$ $z / p_{1 / d}^{\left(q^{d}\right)}\left(1, z^{d}\right)$ and $f_{k}(z)=f^{\left[k, q^{1 / d}\right]}(z)$

$$
f^{0}(z)=\frac{1}{p_{1+1 / d}^{\left(q^{d}\right)}\left(1, q^{-d} z^{d}\right)} \quad \text { and } \quad \tilde{f}_{0}(z)=\frac{1}{1-q^{-d} z^{d}} .
$$

$\left(f_{k}(z)\right.$ of course is an analogue for $z^{k} /\left(1-z^{d}\right)^{k / d}$.) The corresponding Lagrange formulas appear in [12 and 19]. 
7. The $q$-Lagrange inversion formula of Gessel and Stanton, a generalization by Carlitz. Let $\mathbf{K}$ be a field of characteristic zero. Gessel and Stanton's [11] formula essentially is a $q$-analogue of the inverse relations of Gould type [22, p. 52]. As application of our operator method in connection with this Lagrange formula has already been discussed by Hofbauer in [16]. We shall only state the result and leave the details to the reader.

The sequence $\left(f_{k}(z)\right)_{k \in \mathbf{Z}}$ with

$$
f_{k}(z)=\sum_{n \geq k} \frac{\left(A p^{k} q^{k}, p\right)_{n-k}}{(q, q)_{n-k}} q^{\left(\begin{array}{c}
n-k \\
2
\end{array}\right)} z^{n},
$$

where $A, p \in \mathbf{K}$, satisfies the eigenvalue equation

$$
(z+1) \varepsilon^{(q)} f_{k}(z)=q^{k}\left(f_{k}(z)+A z \varepsilon^{(p)} \varepsilon^{(q)} f_{k}(z)\right) .
$$

The sequence $\left(h_{k}(z)\right)_{k \in \mathbf{Z}}$, given by

$$
h_{k}(z)=\sum_{l \leq k} \frac{\left(A p^{k-1} q^{k}, p^{-1}\right)_{k-l}}{(q, q)_{k-l}}(-1)^{k-l} z^{-l},
$$

satisfies the dual eigenvalue equation

$$
\varepsilon^{(1 / q)}(z+1) h_{k}(z)=q^{k}\left(h_{k}(z)+A \varepsilon^{(1 / p)} \varepsilon^{(1 / q)} z h_{k}(z)\right) .
$$

Therefore the wanted sequence $\left(\tilde{f}_{k}(z)\right)_{k \in \mathbf{Z}}$ is, by (4.6),

$$
\tilde{f}_{k}(z)=\sum_{l \leq k} \frac{\left(A p^{k-1} q^{k}, p^{-1}\right)_{k-l-1}}{(q, q)_{k-l}}\left(1-A p^{l} q^{l}\right)(-1)^{k-l} z^{-l} .
$$

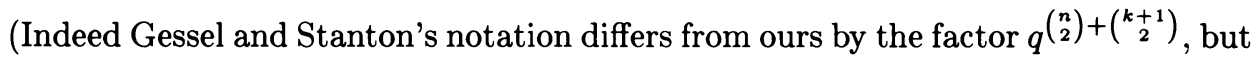
obviously both inverse relations are equivalent. Our choice of constants warrants the condition $f_{k k}=1$ for all $k$.)

If $q=p^{c-1}, A=p^{a}$ and $p \rightarrow 1$, then this inverse relation turns into the classical Gould-type relation if $c \neq 1$ :

$$
f_{n k}=\left(\begin{array}{c}
a+n+c k-k-1 \\
n-k
\end{array}\right)
$$

if and only if

$$
F_{k l}=(-1)^{k-l} \frac{a+c l}{a+c k}\left(\begin{array}{c}
a+c k \\
k-l
\end{array}\right) .
$$

(The factors $(c-1)^{k-n}$ and $(c-1)^{l-k}$ are negligible.) In [14] Gould and Hsu found a generalization of this inverse relation, which can be written as

$$
f_{n k}=\frac{\prod_{j=k+1}^{n}\left(a_{j}+k b_{j}\right)}{(n-k) !}
$$

if and only if

$$
F_{k l}=(-1)^{k-l} \frac{a_{l+1}+l b_{l+1}}{a_{l+1}+k b_{l+1}} \frac{\prod_{j=l+1}^{k}\left(a_{j}+k b_{j}\right)}{(k-l) !}
$$

for arbitrary $a_{j}, b_{j} \in \mathbf{K}$. 
Carlitz [2] gave a $q$-extension of this generalized Gould-type inverse relation which contains the above-stated formula of [11] as a special case. His viewpoint is quite different, though. Whereas Carlitz restricts his interest to inverse relations, Gessel and Stanton apply their formula to derive transformations of basic hypergeometric series, Rogers-Ramanujan identities, etc. The next theorem presents Carlitz's inverse relation in a somewhat modified form.

THEOREM 11 (CARLITZ). Let $a_{j}, b_{j}$ be elements of $\mathbf{K}(q)$. Then

$$
f_{n k}=\frac{\prod_{j=k+1}^{n}\left(a_{j}+q^{k} b_{j}\right)}{(q, q)_{n-k}} q^{\left(\begin{array}{c}
n-k \\
2
\end{array}\right)}
$$

if and only if

$$
F_{k l}=(-1)^{k-l} \frac{a_{l+1}+q^{l} b_{l+1}}{a_{l+1}+q^{k} b_{l+1}} \frac{\prod_{j=l+1}^{k}\left(a_{j}+q^{k} b_{j}\right)}{(q, q)_{k-l}} .
$$

ProOF. To apply Theorem 1 we let $\mathbf{A}=\mathbf{K}(q)$. Set

$$
f_{k}(z)=\sum_{n \geq k} \frac{\prod_{j=k+1}^{n}\left(a_{j}+q^{k} b_{j}\right)}{(q, q)_{n-k}} q^{\left({ }_{2}^{n-k}\right)} z^{n} .
$$

Then

$$
\left(\mathcal{L}\left(\underline{1}, 0,\left(a_{j}\right)_{j \in \mathbf{Z}}\right) z+1\right) \varepsilon^{(q)} f_{k}(z)=q^{k}\left(f_{k}(z)-\mathcal{L}\left(1,0,\left(b_{j}\right)_{j \in \mathbf{Z}}\right) z \varepsilon^{(q)} f_{k}(z)\right),
$$

which we prove by comparing coefficients of $z^{n}$. The dual equation for the auxiliary sequence $\left(h_{k}(z)\right)_{k \in \mathbf{Z}}$ then becomes, from (4.10), (4.18), and because of $\tilde{\underline{1}}=$ $\left(z^{-k}\right)_{k \in \mathbf{Z}}$

$$
\begin{aligned}
& \varepsilon^{(1 / q)}\left(z \mathcal{L}\left(\underline{1}, 0,\left(a_{-j}\right)_{j \in \mathbf{Z}}\right)+1\right) h_{k}(z) \\
& \quad=q^{k}\left(h_{k}(z)-\varepsilon^{(1 / q)} z \mathcal{L}\left(\underline{1}, 0,\left(b_{-j}\right)_{j \in \mathbf{Z}}\right) h_{k}(z)\right) .
\end{aligned}
$$

Comparing coefficients of $z^{-l}$ leads to

$$
q^{l}\left(a_{l+1} h_{k, l+1}+h_{k, l}\right)=q^{k}\left(h_{k l}-q^{l} b_{l+1} h_{k, l+1}\right)
$$

or

$$
h_{k l}=-\frac{a_{l+1}+q^{k} b_{l+1}}{1-q^{k-l}} h_{k, l+1}
$$

from which we obtain

$$
h_{k l}=(-1)^{k-l} \frac{\prod_{j=l+1}^{k}\left(a_{j}+q^{k} b_{j}\right)}{(q, q)_{k-l}}
$$

by setting $h_{k k}=1$. Then a short calculation shows that, by (4.6),

$$
\tilde{f}_{k}(z)=\sum_{l \leq k}(-1)^{k-l} \frac{a_{l+1}+q^{l} b_{l+1}}{a_{l+1}+q^{k} b_{l+1}} \frac{\prod_{j=l+1}^{k}\left(a_{j}+q^{k} b_{j}\right)}{(q, q)_{k-l}} z^{-l},
$$

which completes the proof.

Gessel and Stanton's formula arises from (7.4) by setting $a_{j}=1$ and $b_{j}=$ $-A p^{j-1}$. Their method of proving their formula in [12] also suffices to show (7.4); 
moreover, (7.4) seems to be the most general inverse relation which can be proved by this method.

In [4] Egorychev found two generalizations to several variables for the Gould-type inverse relations for the case $q=1$, namely

$$
\begin{aligned}
f_{n k} & =\left(\begin{array}{c}
a+\sum_{i=1}^{s}\left(n_{i}+c_{i} k_{i}-k_{i}\right)-1 \\
n_{1}-k_{1} \cdots n_{s}-k_{s}
\end{array}\right), \\
F_{k l} & =(-1)^{|k-l|} \frac{a+\sum_{i=1}^{s} c_{i} l_{i}}{a+\sum_{i=1}^{s} c_{i} k_{i}}\left(\begin{array}{c}
a+\sum_{i=1}^{s} c_{i} k_{i} \\
k_{1}-l_{1} \cdots k_{s}-l_{s}
\end{array}\right),
\end{aligned}
$$

and

$$
\begin{aligned}
& f_{n k}=\prod_{j=1}^{s}\left(\begin{array}{c}
a_{j}+c_{j}|n| \\
n_{j}-k_{j}
\end{array}\right) \\
& F_{k l}=(-1)^{|k-l|}\left(1+\sum_{i=1}^{s} \frac{c_{i}\left(k_{i}-l_{i}\right)}{a_{i}+c_{i}|l|}\right) \prod_{j=1}^{s}\left(\begin{array}{c}
a_{j}+c_{j}|l|+k_{j}-l_{j}-1 \\
k_{j}-l_{j}
\end{array}\right)
\end{aligned}
$$

or equivalently

$$
\begin{aligned}
f_{n k} & =\prod_{j=1}^{s}\left(\begin{array}{c}
a_{j}+c_{j}|k|+n_{j}-k_{j}-1 \\
n_{j}-k_{j}
\end{array}\right) \\
F_{k l} & =(-1)^{|k-l|}\left(1-\sum_{i=1}^{s} \frac{c_{i}\left(k_{i}-l_{i}\right)}{a_{i}+c_{i}|k|}\right) \prod_{j=1}^{s}\left(\begin{array}{c}
a_{j}+c_{j}|k| \\
k_{j}-l_{j}
\end{array}\right) .
\end{aligned}
$$

Here $n, k, l$ are multi-indices. The multinomial coefficient

$$
\left(\begin{array}{c}
M \\
N_{1} \cdots N_{s}
\end{array}\right)
$$

in (7.5) means

$$
\frac{M !}{N_{1} ! \cdots N_{s} !\left(M-N_{1}-\cdots-N_{s}\right) !} .
$$

These relations are proved by the Lagrange-Good inversion treated in $\S 5$. For (7.5) we have to observe that for the sequence

$$
f_{k}(z)=\left(\prod_{j=1}^{s} \frac{z_{j}^{k_{j}}}{\left(1-\sum_{i=1}^{s} z_{i}\right)^{c_{j} k_{j}}}\right) \frac{1}{\left(1-\sum_{i=1}^{s} z_{i}\right)^{a}}
$$

the sequence $\tilde{f}_{k}(z)$ by $(5.3)$ is

$$
\tilde{f}_{k}(z)=\left(1+\frac{\sum_{i=1}^{s} c_{i} z_{i}}{1-\sum_{i=1}^{s} z_{i}}\right)\left(\prod_{j=1}^{s} \frac{\left(1-\sum_{i=1}^{s} z_{i}\right)^{c_{j} k_{j}}}{z_{j}^{k_{j}}}\right)\left(1-\sum_{i=1}^{s} z_{i}\right)^{a}
$$

(7.7) corresponds to the pair

$$
f_{k}(z)=\prod_{j=1}^{s} \frac{z_{j}^{k_{j}}}{\left(1-z_{j}\right)^{a_{j}+c_{j}|k|}}
$$


and

$$
\tilde{f}_{k}(z)=\left(1+\sum_{i=1}^{s} \frac{c_{i} z_{i}}{1-z_{i}}\right) \prod_{j=1}^{s} \frac{\left(1-z_{j}\right)^{a_{j}+c_{j}|k|}}{z_{j}^{k_{j}}} .
$$

In (7.6) the roles of $f_{n k}$ and $F_{k l}$ have to be exchanged. Then the relation corresponds to the above pair with the expression $\left(1+\sum_{i=1}^{s}\left(c_{i} z_{i} /\left(1-z_{i}\right)\right)\right)$ shifted from $\tilde{f}_{k}(z)$ to $f_{k}(z)$.

To find a $q$-analogue for the first expression, one might try for $f_{n k}$ the expression

$$
\frac{\left(A p^{|k|} q_{1}^{k_{1}} \cdots q_{s}^{k_{s}}, p\right)_{|n-k|}}{\left(q_{1}, q_{1}\right)_{n_{1}-k_{1}} \cdots\left(q_{s}, q_{s}\right)_{n_{s}-k_{s}}}
$$

times suitable powers of the $q_{i}$. It is not difficult to obtain the missing term either by finding the "right" eigenvalue equations for $f_{k}(z)$ or by extending Gessel's original proof (in [12]) of his one-variable inverse relation to $s$ variables. Once having done this it is easy to extend even the more general Theorem 11 to $s$ variables. The result is

THEOREM 12. Let $a_{j}, b_{j}$ be elements of $\mathbf{K}\left(q_{1}, q_{2}, \ldots, q_{s}\right)$. Then

$$
\left.f_{n k}=\frac{\prod_{j=|k|+1}^{|n|}\left(a_{j}+q_{1}^{k_{1}} \cdots q_{s}^{k_{s}} b_{j}\right)}{\left(q_{1}, q_{1}\right)_{n_{1}-k_{1}} \cdots\left(q_{s}, q_{s}\right)_{n_{s}-k_{s}}} \prod_{i=1}^{s} q_{i}^{\left(n_{i}-k_{i}\right.}\right)-k_{i} \sum_{r=1}^{i-1}\left(n_{r}-k_{r}\right)
$$

if and only if

$$
\begin{array}{r}
F_{k l}=(-1)^{|k-l|} \frac{a_{|l|+1}+q_{1}^{l_{1}} \cdots q_{s}^{l_{s}} b_{|l|+1}}{a_{|l|+1}+q_{1}^{l_{1}} \ldots q_{s}^{l_{s}} b_{|l|+1}} \frac{\prod_{j=|l|+1}^{|k|}\left(a_{j}+q_{1}^{k_{1}} \cdots q_{s}^{k_{s}} b_{j}\right)}{\left(q_{1}, q_{1}\right)_{k_{1}-l_{1}} \cdots\left(q_{s}, q_{s}\right)_{k_{s}-l_{s}}} \prod_{i=1}^{K_{i}} q_{i}^{K_{i}} \\
\text { where } K_{i}=-k_{i} \sum_{r=1}^{i-1}\left(k_{r}-l_{r}\right) .
\end{array}
$$

PROOF. To apply Theorem 1 we need to take $\mathbf{A}=\mathbf{K}\left(q_{1}, \ldots, q_{s}\right)$. Set $f_{k}(z)=$ $\sum_{n \geq k} f_{n k} z^{n}$. For the linear operator $\mathcal{L}\left(\underline{1}, 0,\left(a_{|j|}\right)_{j \in \mathbf{Z}^{*}}\right)$ we write, for short, $\mathcal{A}$ and for $\mathcal{L}\left(\underline{1}, 0,\left(b_{|j|}\right)_{j \in \mathbf{Z}^{*}}\right)$, we write $\mathfrak{B}$. Then

$$
\begin{aligned}
\left(A \sum_{i=1}^{s} z_{i} \varepsilon_{1}^{\left(q_{1}\right)} \cdots \varepsilon_{i}^{\left(q_{i}\right)}\right. & \left.+\varepsilon_{1}^{\left(q_{1}\right)} \cdots \varepsilon_{s}^{\left(q_{s}\right)}\right) f_{k}(z) \\
& =q_{1}^{k_{1}} \cdots q_{s}^{k_{s}}\left(f_{k}(z)-\mathfrak{B} \sum_{i=1}^{s} z_{i} \varepsilon_{1}^{\left(q_{1}\right)} \cdots \varepsilon_{i}^{\left(q_{i}\right)} f_{k}(z)\right),
\end{aligned}
$$

which again is proved by comparing coefficients of $z^{n}$. The dual equation for the sequence $\left(h_{k}(z)\right)_{k \in Z^{*}}$ is then

$$
\begin{aligned}
& \left(\sum_{i=1}^{s} \varepsilon_{1}^{\left(1 / q_{1}\right)} \cdots \varepsilon_{i}^{\left(1 / q_{i}\right)} z_{i} A^{*}+\varepsilon_{1}^{\left(1 / q_{1}\right)} \cdots \varepsilon_{s}^{\left(1 / q_{s}\right)}\right) h_{k}(z) \\
& =q_{1}^{k_{1}} \cdots q_{s}^{k_{s}}\left(h_{k}(z)-\sum_{i=1}^{s} \varepsilon_{1}^{\left(1 / q_{1}\right)} \cdots \varepsilon_{i}^{\left(1 / q_{i}\right)} z_{i} \mathfrak{B}^{*}\right) h_{k}(z) .
\end{aligned}
$$


Since by (4.18), $A^{*} z^{-l}=a_{|l|} z^{-l}$ and $\mathfrak{B}^{*} z^{-l}=b_{|l|} z^{-l}$, comparing the coefficients of $z^{-l}$ leads to a recursion relation for the coefficients of $h_{k}(z)$ which finally gives

$$
h_{k l}=(-1)^{|k-l|} \frac{\prod_{j=|l|+1}^{|k|}\left(a_{j}+q_{1}^{k_{1}} \cdots q_{s}^{k_{s}} b_{j}\right)}{\left(q_{1}, q_{1}\right)_{k_{1}-l_{1}} \cdots\left(q_{s}, q_{s}\right)_{k_{s}-l_{s}}} \prod_{i=1}^{s} q_{i}^{K_{i}} .
$$

By (4.6) we get, after a short calculation,

$$
\begin{aligned}
\tilde{f}_{k}(z)= & \sum_{l \leq k}(-1)^{|k-l|} \frac{a_{|l|+1} q_{1}^{l_{1}} \cdots q^{l_{s}} b_{|l|+1}}{a_{|l|+1}+q_{1}^{k_{1}} \cdots q^{k_{s}} b_{|l|+1}} \\
& \cdot \frac{\prod_{j=|l|+1}^{|k|}\left(a_{j}+q_{1}^{k_{1}} \cdots q_{s}^{k_{s}} b_{j}\right)}{\left(q_{1}, q_{1}\right)_{k_{1}-l_{1}} \cdots\left(q_{s}, q_{s}\right)_{k_{s}-l_{s}}} \prod_{i=1}^{s} q_{i}^{K_{i}} z^{-l}
\end{aligned}
$$

for $K_{i}=-k_{i} \sum_{r=1}^{l-1}\left(k_{r}-l_{r}\right)$, as desired.

REMARK. We did not mention specifically that it is not self-evident that application of Theorem $1(\mathrm{~A})$ to (7.9) is possible. What has to be checked is condition (4.3). In this case this is $q_{1}^{k_{1}} \cdots q_{s}^{k_{s}}=q_{1}^{n_{1}} \cdots q_{s}^{n_{s}}$ if and only if $n=k$. A single equation is enough information to compute $\tilde{f}_{k}(z)$ by Theorem 1 , although $f_{k}(z)$ is an $s$-variable fps. Normally when dealing with $s$ variables we need $s$ equations, such as in $\S 5$ (yet it is not difficult to add to (7.9) $(s-1)$ similar equations).

The special case for $b_{j}=-A p^{j}$ and $a_{j}=1$ is the $q$-analogue of (7.5).

COROLLARY 13. The following inverse relation holds:

$$
f_{n k}=\frac{\left(A p^{|k|} q_{1}^{k_{1}} \cdots q_{s}^{k_{s}}, p\right)_{|n-k|}}{\left(q_{1}, q_{1}\right)_{n_{1}-k_{1}} \cdots\left(q_{s}, q_{s}\right)_{n_{s}-k_{s}}} \prod_{i=1}^{s} q_{i}^{K_{i}^{\prime}}
$$

where

$$
K_{i}^{\prime}=\left(\begin{array}{c}
n_{i}-k_{i} \\
2
\end{array}\right)-k_{i} \sum_{r=1}^{i-1}\left(n_{r}-k_{r}\right)
$$

if and only if

$$
\begin{aligned}
F_{k l}= & (-1)^{|k-l|} \frac{\left(1-A p^{|l|} q^{l_{1}} \cdots q_{s}^{l_{s}}\right)}{\left(1-A p^{|l|} q_{1}^{k_{1}} \cdots q_{s}^{k_{s}}\right)} \\
& \cdot \frac{\left(A p^{|k|-1} q_{1}^{k_{1}} \cdots q_{s}^{k_{s}}, p^{-1}\right)_{|k-l|}}{\left(q_{1}, q_{1}\right)_{k_{1}-l_{1}} \cdots\left(q_{s}, q_{s}\right)_{k_{s}-l_{s}}} \prod_{i=1}^{K_{1}} q_{1}^{K_{i}}
\end{aligned}
$$

By setting $q_{i}=p^{c_{i}-1}, A=p^{a}$ and $p \rightarrow 1$, we get the inverse relation (7.5) (again after deleting the factors $\left(c_{1}-1\right)^{k_{n}-n_{1}} \cdots\left(c_{s}-1\right)^{k_{s}-n_{s}}$ and $\left(c_{1}-1\right)^{l_{1}-k_{1}} \ldots$ $\left.\left(c_{s}-1\right)^{l_{s}-k_{s}}\right)$.

Concluding, we remark that the inversion formulas of [12] are proved in a similar manner.

8. The author's $q$-analogue. This section deals with the $q$-analogue given in $[15,18,19]$. Here we do not have such an extensive theory as in $\S 6$, but there are many examples, which yield $q$-extensions of Riordan's inverse relations (see [19]), special polynomials, etc. Let $s=1$ and $\mathbf{A}=\mathbf{K}(q)$ for a field $\mathbf{K}$. 
The essential definition is

DEFinition 14. The fps $\varphi_{\alpha}(z), \alpha \in \mathbf{R}$, are called $q$-powers for a fixed fps $\varphi(z)$ if $\varphi_{\alpha}(0) \neq 0$ for all $\alpha$ and

$$
D^{(q)} \varphi_{\alpha}(z)=[\alpha] \varphi(z) \varphi_{\alpha}(z) .
$$

Obviously in the case $q=1$ the fps $\varphi_{\alpha}(z)$ (save a constant) are powers of an fps $\bar{\varphi}(z)$ with $\varphi(z)=\bar{\varphi}^{\prime}(z) / \bar{\varphi}(z)$. If we write $(8.1)$ as

$$
\varepsilon^{(q)} \varphi_{\alpha}(z)=\left(1+\left(q^{\alpha}-1\right) z \varphi(z)\right) \varphi_{\alpha}(z),
$$

we obtain, by successive use of this formula,

$$
\varphi_{\alpha}(z)=\frac{1}{\prod_{j=0}^{\infty}\left(1+\left(q^{\alpha}-1\right) q^{j} z \varphi\left(q^{j} z\right)\right)},
$$

or, by Garsia's notion of starring [7],

$$
\varphi_{\alpha}(z)=\left(\frac{1}{1+\left(q^{\alpha}-1\right) z \varphi(z)}\right)^{*} .
$$

A short evaluation shows that for $a, b \in \mathbf{R}(\mathbf{C})$ and $m \in \mathbf{N}$,

EXAMPLE 15. $e_{q^{m}}\left((a[\alpha]+b) z^{m}\right) / e_{q^{m}}\left(b z^{m}\right)$ are $q$-powers corresponding to

$$
\varphi(z)=a[m] z^{m-1} /\left(1+\left(q^{m}-1\right) b z^{m}\right) .
$$

This is the most general known example for $q$-powers in the sense of Definition 14, but it suffices for the applications.

Let $\varphi_{\alpha}(z)$ and $\Phi_{\alpha}(z)$. be $q$-powers for $\varphi(z)$ and $\Phi(z)$, respectively. Then we consider the sequence $\mathfrak{f}=\left(f_{k}(z)\right)_{k \in \mathbf{Z}}$, where

$$
f_{k}(z)=\frac{z^{k}}{\varphi_{k+\lambda}(q z) / \Phi_{-k-\mu}(z)} .
$$

By (8.2) we get for $f_{k}(z)$,

$$
\varepsilon^{(q)} f_{k}(z)=q^{k} \frac{1+\left(q^{-k-\mu}-1\right) z \Phi(z)}{\left(1+\left(q^{k+\lambda}-1\right) q z \varphi(q z)\right)} f_{k}(z),
$$

and, after a short calculation,

$$
\left(\varepsilon^{(q)}(1-z \varphi(z))-q^{-\mu} z \Phi(z)\right) f_{k}(z)=q^{k}\left(-q^{\lambda} \varepsilon^{(q)} z \varphi(z)+1-z \Phi(z)\right) f_{k}(z)
$$

Thus the dual equation for the auxiliary sequence $\left(h_{k}(z)\right)_{k \in \mathbf{Z}}$, by $(4.4)$, is

$$
\left((1-z \varphi(z)) \varepsilon^{(1 / q)}-q^{-\mu} z \Phi(z)\right) h_{k}(z)=q^{k}\left(-q^{\lambda} z \varphi(z) \varepsilon^{(1 / q)}+1-z \Phi(z)\right) h_{k}(z)
$$
or, equivalently,

$$
\varepsilon^{(1 / q)} h_{k}(z)=q^{k} \frac{1+\left(q^{-k-\mu}-1\right) z \Phi(z)}{1+\left(q^{k+\lambda}-1\right) z \varphi(z)} h_{k}(z)
$$

then finally

$$
\varepsilon^{(q)} h_{k}(z)=q^{-k} \frac{1+\left(q^{k+\lambda}-1\right) q z \varphi(q z)}{1+\left(q^{-k-\mu}-1\right) q z \Phi(q z)} h_{k}(z)
$$

Thus

$$
h_{k}(z)=\frac{\varphi_{k+\lambda}(q z) / \Phi_{-k-\mu}(q z)}{z^{k}}
$$


and, by (4.6),

$$
\begin{aligned}
\tilde{f}_{k}(z)= & \left(-q^{\lambda} z \varphi(z) \varepsilon^{(1 / q)}+1-z \Phi(z)\right) h_{k}(z) \\
= & \left(-q^{k+\lambda} z \varphi(z)\left(1+\left(q^{-k-\mu}-1\right) z \Phi(z)\right)\right. \\
& \left.+(1-z \Phi(z))\left(1+\left(q^{k+\lambda}-1\right) z \varphi(z)\right)\right) \frac{\varphi_{k+\lambda}(z) / \Phi_{-k-\mu}(q z)}{z^{k}} \\
= & \left(1-z \varphi(z)-z \Phi(z)+\left(1-q^{\lambda-\mu}\right) z^{2} \varphi(z) \Phi(z)\right) \frac{\varphi_{k+\lambda}(z) / \Phi_{-k-\mu}(q z)}{z^{k}} .
\end{aligned}
$$

The necessary condition $\left\langle f_{k}(z), \tilde{f}_{k}(z)\right\rangle=1$ is easily verified. Thus we get the Lagrange formula

THEOREM 16. The coefficients in the expansion

$$
g(z)=\sum_{k \in \mathbf{Z}} c_{k} \frac{z^{k}}{\varphi_{k+\lambda}(q z) / \Phi_{-k-\mu}(z)}
$$

with $g(z) \in \overline{L s}(z), \varphi_{\alpha}(z)$ and $\Phi_{\alpha}(z)$ being $q$-powers for $\varphi(z)$ and $\Phi(z)$, respectively, are given by

$$
\begin{aligned}
c_{n}= & \left\langle g(z),\left(1-z \varphi(z)-z \Phi(z)+\left(1-q^{\lambda-\mu}\right) z^{2} \varphi(z) \Phi(z)\right)\right. \\
= & \left\langle z^{0}\right\rangle g(z)\left(1-z \varphi(z)-z \Phi(z)+\left(1-q^{\lambda-\mu}\right) z^{2} \varphi(z) \Phi(z)\right) \\
& \cdot \frac{\varphi_{n+\lambda}(z) / \Phi_{-n-\mu}(q z)}{z^{n}} \cdot \text {. }
\end{aligned}
$$

In [18] also an analogue of the first version (1.1) of the Lagrange formula is obtained. This shall be generalized here. First recall the case $q=1$. There the expansion (8.10) corresponds to an expansion of the form

$$
g(z)=\sum_{k \in \mathbf{Z}} \frac{c_{k} f^{k}(z)}{a(z)}
$$

if we assume $\lim _{q \rightarrow 1} \varphi_{\alpha}(z)=\bar{\varphi}^{\alpha}(z), \lim _{q \rightarrow 1} \Phi_{\alpha}(z)=\bar{\Phi}^{\alpha}(z), z / \bar{\varphi}(z) \bar{\Phi}(z)=f(z)$ and $\bar{\Phi}^{\mu}(z) \bar{\varphi}^{\lambda}(z)=a(z)$. Then by $(1.1)$,

$$
\dot{c_{n}}=\frac{1}{n}\left\langle z^{-1}\right\rangle(g(z) \cdot a(z))^{\prime} f^{-n}(z) \text { for } n \neq 0
$$

The $q$-analogue of this formula is

THEOREM 17. The coefficients in the expansion

$$
g(z)=\sum_{k \in \mathbf{Z}} c_{k} \frac{z^{k}}{\varphi_{k+\lambda}(z) / \Phi_{-k-\mu}(z)},
$$

with the assumptions of Theorem 1 , for $n \neq 0$ are given by

$$
c_{n}=\frac{1}{[n]}\left\langle z^{-1}\right\rangle D^{(q)}\left(\frac{g(z) \varphi_{\lambda}(z)}{\Phi_{-\mu}(z)}\right) \frac{\varphi_{n+\lambda}(z) / \Phi_{-n-\mu}(q z)}{z^{n} \varphi_{\lambda}(z) / \Phi_{-\mu}(q z)} .
$$


ProOF. This time we start with the sequence $f=\left(f_{k}(z)\right)_{k \in \mathbf{Z}}$, where

$$
f_{k}(z)=\frac{z^{k} \varphi_{\lambda}(z) / \Phi_{-\mu}(z)}{\varphi_{k+\lambda}(z) / \Phi_{-k-\mu}(z)}
$$

By (8.2) we get, after a short calculation (analogous to that which yielded (8.6)),

$$
\begin{aligned}
& \left(\frac{1-z \varphi(z)}{1+\left(q^{\lambda}-1\right) z \varphi(z)} \varepsilon^{(q)}-\frac{q^{-\mu} z \Phi(z)}{1+\left(q^{-\mu}-1\right) z \Phi(z)}\right) f_{k}(z) \\
& \quad=q^{k}\left(-\frac{q^{\lambda} z \varphi(z)}{1+\left(q^{\lambda}-1\right) z \varphi(z)} \varepsilon^{(q)}+\frac{1-z \Phi(z)}{1+\left(q^{-\mu}-1\right) z \Phi(z)}\right) f_{k}(z)
\end{aligned}
$$

which is equivalent to

$$
z D^{(q)} f_{k}(z)=[k]\left(-\frac{q^{\lambda} z \varphi(z)}{1+\left(q^{\lambda}-1\right) z \varphi(z)} \varepsilon^{(q)}+\frac{1-z \Phi(z)}{1+\left(q^{-\mu}-1\right) z \Phi(z)}\right) f_{k}(z)
$$

Thus the dual equation for the sequence $\left(h_{k}(z)\right)_{k \in \mathbf{Z}}$, remembering $(4.10)$, is

$$
\begin{aligned}
& -\frac{1}{q} z D^{(1 / q)} h_{k}(z) \\
& \quad=[k]\left(-\varepsilon^{(1 / q)} \frac{q^{\lambda} z \varphi(z)}{1+\left(q^{\lambda}-1\right) z \varphi(z)}+\frac{1-z \Phi(z)}{1+\left(q^{-\mu}-1\right) z \Phi(z)}\right) h_{k}(z)
\end{aligned}
$$

which finally leads to (quite analogously to the considerations which proved (8.8))

$$
h_{k}(z)=\frac{\varphi_{k+\lambda}(z) / \Phi_{-k-\mu}(q z)}{z^{k} \varphi_{\lambda}(z) / \Phi_{-\mu}(q z)} .
$$

Therefore by Corollary 2 we get, by letting $U=z D^{(q)}, g(k)=[k]$, after having compared (8.15) and (4.2):

The coefficients in the expansion

$$
\bar{g}(z)=\sum_{k \in \mathbf{Z}} c_{k} \frac{z^{k} \varphi_{\lambda}(z) / \Phi_{-\mu}(z)}{\varphi_{k+\lambda}(z) / \Phi_{-k-\mu}(z)}
$$

are given by (for $n \neq 0$ )

$$
c_{n}=\frac{1}{[n]}\left\langle z D^{(q)} \bar{g}(z), \frac{\varphi_{n+\lambda}(z) / \Phi_{-k-\mu}(q z)}{z^{n} \varphi_{\lambda}(z) / \Phi_{-\mu}(q z)}\right\rangle .
$$

But after multiplying both sides of $(8.12)$ by $\varphi_{\lambda}(z) / \Phi_{-\mu}(z)$, we see that this is equivalent to (8.13).

REMARK. (1) In [18] only the special case $\lambda=\mu=0$ is proved.

(2) Indeed here we have analogues of both types of the Lagrange formula. For lack of identities similar to (6.1)-(6.4) this theory seemingly cannot be further developed. In particular we are not able to say something reasonable about the inverse sequence $\mathfrak{F}$ in contrast to Garsia's theory. (Via (8.15) and Theorem 5 we could get a recursive formula for the $F_{l}(z)$. It is omitted here, because it is usable only in special cases.) The reason is that the fps $\varphi(z)$ seems to be the only connection between the powers $\varphi_{\alpha}(z)$. 
EXAMPLE 18. This example should demonstrate use of our Theorem 16 and 17. It concerns the problem of finding $q$-analogues of Euler's [5] formula

$$
e^{b z}=\sum_{n=0}^{\infty} \frac{b(b+n)^{n-1}}{n !}\left(z e^{-z}\right)^{n} .
$$

An equivalent form of $(8.18)$ is

$$
1=\sum_{n=0}^{\infty} \frac{b(b+n)^{n-1}}{n !} \frac{z^{n}}{e^{(b+n) z}}
$$

A reasonable analogue for $e^{n z}$, or better $e^{(b+n) z} / e^{b z}$, in view of Example 15, is $e_{1 / q}\left(\left(b+q^{-1}[n]_{1 / q}\right) z\right) / e_{1 / q}(b z)$. In Example $15 q$ has to be replaced by $1 / q$, and $a$ is set equal to $q^{-1}$. Then these fps turn out to be $1 / q$-powers corresponding to $\varphi(z)=1 / q(1+(q-1) b z)$. (The choice of the base $1 / q$ instead of $q$ will be explained later.) Theorem 17 for $\lambda=\mu=0, \Phi(z)=0$ and $q$ replaced by $1 / q$ gives: If

$$
e_{1 / q}(b z)=\sum_{k \in \mathbf{Z}} c_{k} \frac{z^{k}}{e_{1 / q}\left(\left(b+q^{-1}[k]_{1 / q}\right) z\right) / e_{1 / q}(b z)}
$$

then for $n \geq 1$,

$$
\begin{aligned}
c_{n} & =\frac{1}{[n]_{1 / q}}\left\langle z^{-1}\right\rangle D^{(1 / q)}\left(e_{1 / q}(b z)\right) \cdot \frac{e_{1 / q}\left(\left(b+q^{-1}[n]_{1 / q}\right) z\right) / e_{1 / q}(b z)}{z^{n}} \\
& =\frac{1}{[n]_{1 / q}}\left\langle z^{-1}\right\rangle \frac{b e_{1 / q}\left(\left(b+q^{-1}[n]_{1 / q}\right) z\right)}{z^{n}} \\
& =q^{\left(\begin{array}{c}
n \\
2
\end{array}\right)} \frac{b}{[n] !}\left(b+q^{-1}[n]_{1 / q}\right)^{n-1} .
\end{aligned}
$$

Of course $c_{0}=1$ and $c_{n}=0$ for $n$ negative. The desired analogue for (8.19) is therefore

$$
1=\sum_{n=0}^{\infty} \frac{q^{\left(\begin{array}{c}
n \\
2
\end{array}\right)}}{[n] !} b\left(b+q^{-n}[n]\right)^{n-1} \frac{z^{n}}{e_{1 / q}\left(\left(b+q^{-n}[n] z\right)\right)} .
$$

The similar formula

$$
\frac{1}{1-z(1+(q-1) b)}=\sum_{n=0}^{\infty} \frac{q^{\left(\begin{array}{c}
n+1 \\
2
\end{array}\right)}}{[n] !}\left(b+q^{-n}[n]\right)^{n} \frac{z^{n}}{e_{1 / q}\left(\left(b+q^{-n}[n]\right) z\right)},
$$

which is easily proved by Theorem 16 , is a $q$-analogue for the well-known identity

$$
\frac{1}{1-z}=\sum_{n=0}^{\infty} \frac{(b+n)^{n}}{n !} \frac{z^{n}}{e^{(b+n) z}}
$$

If we multiply $(8.21)$ by $1-z(1+(q-1) b)$ and then add to $(8.20)$, we obtain

$$
\begin{aligned}
2=\sum_{n=0}^{\infty} \frac{q^{\left(\begin{array}{c}
n \\
2
\end{array}\right)}}{[n] !}\left(b+q^{-n}[n]\right)^{n-1}\left(((1-z)+z(1-q) b)\left(q^{n} b+[n]\right)+b\right) \\
\cdot \frac{z^{n}}{e_{1 / q}\left(\left(b+q^{-n}[n]\right) z\right)}
\end{aligned}
$$


which is valid in the sense of fps. In [1, identity (7.48)] Askey and Ismail evaluated the right side of (8.23) if $0<q<1, b \geq 0$ and $z=1$. The result is a curious $q$-analogue for the special case of (8.19), where $z=1$ :

$$
1=\sum_{n=0}^{\infty} \frac{q^{\left(\begin{array}{c}
n \\
2
\end{array}\right)}}{[n] !} b\left(b+q^{-n}[n]\right)^{n-1}\left(2-q^{n}+b(1-q) q^{n}\right) \frac{1}{e_{1 / q}\left(b+q^{-n}[n]\right)} .
$$

$\left(e_{1 / q}(z)\right.$ converges for all $z \in \mathbf{C}$ if $|q|<1$; therefore we took $1 / q$ instead of $q$.) Since the right sides of (8.20) and (8.21) turn out to be analytic functions for $\operatorname{Re} z>0$ because there the series uniformly converge, two questions arise:

(1) Is it possible to evaluate the right sides of $(8.20)$ and (8.21) for $0<q<1$ or even $|q|<1$ ? (Both (8.20) and (8.21) are wrong in this case.)

(2) What is the relationship between (8.24) and $q$-Lagrange inversion? (Askey and Ismail obtain (8.24) in connection with an orthogonality relation for $q$-CarlitzKarlin-McGregor polynomials.)

What can be shown is that (8.20) and (8.21) (and therefore (8.23)) hold for $q>1$ and $|z|<1 /|1+(q-1) b|$ after $e_{1 / q}\left(\left(b+q^{-n}[n]\right) z\right)^{-1}$ is replaced by $e_{q}\left(-\left(b+q^{-n}[n]\right) z\right)$. (In the fps-sense both expressions are identical, but for $q>1$ only the second converges for all $n \in \mathbf{N}$ and $z \in \mathbf{C}$.) This is proved by proving uniform convergence of the right sides of (8.20) and (8.21) for $|z| \leq r, r$ being fixed with $0<r<$ $1 /|1+(q-1) b|$, and using Weierstrass's double series theorem.

For $0<q<1$ this argument does not work, because there does not even exist a neighborhood of 0 where for all $n$ the functions $z^{n} / e_{1 / q}\left(\left(b+q^{-n}[n]\right) z\right)$ are analytic.

After this excursion we turn to the problem of finding $q$-analogues of the $s$ variable Lagrange-Good formula. At first sight this seems to be easily established by suitable extensions of the $q$-powers $\varphi_{\alpha}(z)$ to $s$ variables. But even for $s=2$ great difficulties arise. Still we succeed in establishing some special two-dimensional formulas.

LEMMA 19. Let $\varphi_{\alpha}\left(z_{1}\right)$ and $\Phi_{\alpha}\left(z_{2}\right)$ be $q$-powers for $\varphi\left(z_{1}\right)$ and $\Phi\left(z_{2}\right)$, respectively. Then

(1)

$$
f_{k_{1}, k_{2}}\left(z_{1}, z_{2}\right)=\frac{z_{1}^{k_{1}} z_{2}^{k_{2}}}{\varphi_{k_{1}+k_{2}+\lambda}\left(q z_{1}\right) / \Phi_{-k_{1}-k_{2}-\mu}\left(z_{2}\right)}
$$

satisfies the system

(I) $\quad\left(q^{-\lambda}\left(1-z_{2} \Phi\left(z_{2}\right)\right)-\varepsilon_{1}^{(q)} \varepsilon_{2}^{(q)} z_{1} \varphi\left(z_{1}\right)\right) f_{k_{1}, k_{2}}\left(z_{1}, z_{2}\right)$

$$
\begin{aligned}
= & q^{-k_{1}-\lambda} \varepsilon_{1}^{(q)}\left(1-z_{1} \varphi\left(z_{1}\right)-z_{2} \Phi\left(z_{2}\right)+z_{1} \varphi\left(z_{1}\right) z_{2} \Phi\left(z_{2}\right)\left(1-q^{\lambda-\mu}\right)\right) \\
& \cdot f_{k_{1}, k_{2}}\left(z_{1}, z_{2}\right) ;
\end{aligned}
$$

$$
\begin{aligned}
\left(-q^{-\mu}\right. & \left.z_{2} \Phi\left(z_{2}\right)+\varepsilon_{1}^{(q)} \varepsilon_{2}^{(q)}\left(1-z_{1} \varphi\left(z_{1}\right)\right)\right) f_{k_{1}, k_{2}}\left(z_{1}, z_{2}\right) \\
= & q^{k_{2}} \varepsilon_{1}^{(q)}\left(1-z_{1} \varphi\left(z_{1}\right)-z_{2} \Phi\left(z_{2}\right)+z_{1} \varphi\left(z_{1}\right) z_{2} \Phi\left(z_{2}\right)\left(1-q^{\lambda-\mu}\right)\right) \\
& \cdot f_{k_{1}, k_{2}}\left(z_{1}, z_{2}\right)
\end{aligned}
$$

$$
h_{k_{1}, k_{2}}\left(z_{1}, z_{2}\right)=\frac{\varphi_{k_{1}+k_{2}+\lambda}\left(q z_{1}\right) / \Phi_{-k_{1}-k_{2}-\mu}\left(q z_{2}\right)}{z_{1}^{k_{1}} z_{2}^{k_{2}}}
$$


satisfies the dual system

$$
\begin{gathered}
\left(\mathrm{I}^{*}\right) \quad\left(q^{-\lambda}\left(1-z_{2} \Phi\left(z_{2}\right)\right)-z_{1} \varphi\left(z_{1}\right) \varepsilon_{1}^{(1 / q)} \varepsilon_{2}^{(1 / q)}\right) h_{k_{1}, k_{2}}\left(z_{1}, z_{2}\right) \\
=q^{-k_{1}-\lambda}\left(1-z_{1} \varphi\left(z_{1}\right)-z_{2} \Phi\left(z_{2}\right)+z_{1} \varphi\left(z_{1}\right) z_{2} \Phi\left(z_{2}\right)\left(1-q^{\lambda-\mu}\right)\right) \\
\cdot \varepsilon_{1}^{(1 / q)} h_{k_{1}, k_{2}}\left(z_{1}, z_{2}\right) ; \\
\left(\mathrm{II}^{*}\right) \quad\left(-q^{-\mu} z_{2} \Phi\left(z_{2}\right)+\left(1-z_{1} \varphi\left(z_{1}\right)\right) \varepsilon_{1}^{(1 / q)} \varepsilon_{2}^{(1 / q)}\right) h_{k_{1}, k_{2}}\left(z_{1}, z_{2}\right) \\
=q^{k_{2}}\left(1-z_{1} \varphi\left(z_{1}\right)-z_{2} \Phi\left(z_{2}\right)+z_{1} \varphi\left(z_{1}\right) z_{2} \Phi\left(z_{2}\right)\left(1-q^{\lambda-\mu}\right)\right) \\
\cdot \varepsilon_{1}^{(1 / q)} h_{k_{1}, k_{2}}\left(z_{1}, z_{2}\right) .
\end{gathered}
$$

(3) $B y(4.6)$,

$$
\begin{aligned}
\tilde{f}_{k_{1}, k_{2}}\left(z_{1}, z_{2}\right)= & \left(1-z_{1} \varphi\left(z_{1}\right)-z_{2} \varphi\left(z_{2}\right)+z_{1} \varphi\left(z_{1}\right) z_{2} \Phi\left(z_{2}\right)\left(1-q^{\lambda-\mu}\right)\right) \\
& \cdot \frac{\varphi_{k_{1}+k_{2}+\lambda}\left(z_{1}\right) / \Phi_{-k_{1}-k_{2}-\mu}\left(q z_{2}\right)}{z_{1}^{k_{1}} z_{2}^{k_{2}}} \cdot
\end{aligned}
$$

The proof of this lemma is straightforward.

Equation (4.8) then gives

THEOREM 20. With the assumptions of Lemma 19 and $g\left(z_{1}, z_{2}\right) \in \overline{L s}\left(z_{1}, z_{2}\right)$, the coefficients in the expansion

$$
g\left(z_{1}, z_{2}\right)=\sum_{k \in \mathbf{Z}^{2}} c_{k} \frac{z_{1}^{k_{1}} z_{2}^{k_{2}}}{\varphi_{k_{1}+k_{2}+\lambda}\left(q z_{1}\right) / \Phi_{-k_{1}-k_{2}-\mu}\left(z_{2}\right)}
$$

are computed by

$$
\begin{aligned}
c_{n}= & \left\langle z_{0}\right\rangle g\left(z_{1}, z_{2}\right)\left(1-z_{1} \varphi\left(z_{1}\right)-z_{2} \Phi\left(z_{2}\right)+z_{1} \varphi\left(z_{1}\right) z_{2} \Phi\left(z_{2}\right)\left(1-q^{\lambda-\mu}\right)\right) \\
& . \frac{\varphi_{n_{1}+n_{2}+\lambda}\left(z_{1}\right) / \Phi_{-n_{1}-n_{2}-\mu}\left(q z_{2}\right)}{z_{1}^{n_{1}} z_{2}^{n_{2}}} \cdot
\end{aligned}
$$

Obviously this theorem intimately corresponds to Theorem 16. Indeed the proof of the orthogonality relation in [18, Lemma 1] is easily converted to obtain the orthogonality relation which proves Theorem 20 by $q$-differentiation with respect to $z_{1}$ and $z_{2}$ at the same time (meaning the operator $D_{z_{1}, z_{2}}^{(q)}=\left(\varepsilon_{1}^{(q)} \varepsilon_{2}^{(q)}-1\right) /(q-1)$ ).

EXAMPLE 21. This short application of Theorem 20 concerns the $q$-Catalan numbers treated by MacMahon $[20$, p. 214; 21, pp. 1345, 1429] and Fürlinger and Hofbauer [6]. They are able to compute a generating function of those numbers $C_{n}^{(q)}(x)$ :

$$
z=\sum_{n=1}^{\infty} \frac{q^{-\left(\begin{array}{c}
n \\
2
\end{array}\right)} C_{n}^{(q)}(x) z^{n}}{\left(-q^{-n} z, q\right)_{n}(-q x z, q)_{n}} .
$$

If we write $C_{n}^{(q)}(x)=\sum_{k} r_{n k}(q) x^{k}$, the coefficients $r_{n k}(q)$ are $q$-Runyon numbers. We compute them by Theorem 20 . It is convenient to first set $z=z_{2}$ and $x=z_{1} / z_{2}$. Then (8.25) becomes, by (6.35),

$$
\begin{aligned}
z_{2} & =\sum_{n=1}^{\infty} \frac{q^{-\left(\begin{array}{c}
n \\
2
\end{array}\right)} \sum_{k} r_{n k}(q) z_{1}^{k} z_{2}^{n-k}}{p_{n}^{(q)}\left(1,-q^{-n} z_{2}\right) p_{n}^{(q)}\left(1,-q z_{1}\right)} \\
& =\sum_{k_{1}, k_{2}} \frac{q^{\kappa} r_{k_{1}+k_{2}, k_{1}}(q) z_{1}^{k_{1}} z_{2}^{k_{2}}}{p_{k_{1}+k_{2}}^{(q)}\left(1,-q z_{1}\right) p_{k_{1}+k_{2}}^{(q)}\left(1,-q^{-k_{1}-k_{2}} z_{2}\right)}
\end{aligned}
$$


where

$$
\kappa=-\left(\begin{array}{c}
k_{1}+k_{2} \\
2
\end{array}\right)
$$

According to Example 15, $p_{\alpha}^{(q)}(1,-z)$ are $q$-powers for $1 /(1+z)$ if we set $a=1, b=$ $-1 /(1-q)$ and $m=1$. By setting $\varphi_{\alpha}^{(q)}\left(z_{1}\right)=p_{\alpha}^{(q)}\left(1,-z_{1}\right)$ and $\Phi_{\alpha}(z)=p_{\alpha}^{(q)}\left(1,-z_{2}\right)$ and applying Theorem 20 to (8.26), we get $(\lambda=\mu=0)$

$$
\begin{aligned}
q^{\kappa} r_{k_{1}+k_{2}, k_{1}}(q)= & \left\langle z_{0}\right\rangle z_{2}\left(1-\frac{z_{1}}{1+z_{1}}-\frac{z_{2}}{1+z_{2}}\right) \\
& \cdot \frac{p_{k_{1}+k_{2}}^{(q)}\left(1,-z_{1}\right) / p_{k_{1}+k_{2}}^{(q)}\left(\left(1,-q^{-k_{1}-k_{2}+1} z_{2}\right)\right)}{z_{1}^{k_{1}} z_{2}^{k_{2}}} \\
= & \left\langle z_{0}\right\rangle \frac{\left(1-z_{1} z_{2}\right)}{z_{1}^{k_{1}} z_{2}^{k_{2}-1}} \frac{p_{k_{1}+k_{2}-1}^{(q)}\left(1,-q z_{1}\right)}{p_{k_{1}+k_{2}-1}^{(q)}\left(1,-q^{-k_{1}-k_{2}+1} z_{2}\right)} .
\end{aligned}
$$

After setting $n=k_{1}+k_{2}, k=k_{1}$, we obtain, by (6.35),

$$
\begin{aligned}
q^{-\left(\begin{array}{c}
n \\
2
\end{array}\right)} r_{n k}(q)= & q^{\left(\begin{array}{c}
k+1 \\
2
\end{array}\right)+\left(\begin{array}{c}
n-k \\
2
\end{array}\right)-n(n-k-1)}\left[\begin{array}{c}
n-1 \\
k
\end{array}\right]\left[\begin{array}{c}
n-1 \\
n-k-1
\end{array}\right] \\
& -q^{\left(\begin{array}{c}
k \\
2
\end{array}\right)+\left(\begin{array}{c}
n-k-1 \\
2
\end{array}\right)-n(n-k-2)}\left[\begin{array}{c}
n-1 \\
k-1
\end{array}\right]\left[\begin{array}{c}
n-1 \\
n-k-2
\end{array}\right] \\
= & q^{-\left(\begin{array}{c}
n \\
2
\end{array}\right)+k^{2}+k} \frac{[n-1] !^{2}}{[k] ![n-k] ![k+1] ![n-k-1] !} \\
& \cdot([n-k][k+1]-q[k][n-k-1]) \\
= & q^{-\left(\begin{array}{c}
n \\
2
\end{array}\right)+k^{2}+k} \frac{[n-1] !^{2}}{[k] ![n-k] ![k+1] ![n-k-1] !}[n]
\end{aligned}
$$

and finally

$$
r_{n k}(q)=q^{k^{2}+k} \frac{1}{[n]}\left[\begin{array}{l}
n \\
k
\end{array}\right]\left[\begin{array}{c}
n \\
k+1
\end{array}\right] .
$$

Two further two-dimensional $q$-Lagrange formulas are stated below. The proofs are quite similar to that of Theorem 20. A more detailed discussion of twodimensional $q$-Lagrange formulas is the object of another paper.

THEOREM 22. With $\varphi_{\alpha}(z)$ and $\Phi_{\alpha}(z)$ being $q$-powers for $\varphi(z)$ and $\Phi(z)$, the coefficients in the expansion

$$
g(z)=\sum_{k \in \mathbf{Z}_{2}} c_{k} \frac{z_{1}^{k_{1}} z_{2}^{k_{2}}}{\varphi_{\left(k_{1}+k_{2}\right) / 2+\lambda}\left(q z_{1} z_{2}\right) / \Phi_{-\left(k_{1}+k_{2}\right) / 2-\mu}\left(z_{1} z_{2}\right)}
$$

are given by

$$
\begin{aligned}
c_{n}=\left\langle z^{0}\right\rangle g(z)\left(1-z_{1} z_{2} \varphi\left(z_{1} z_{2}\right)-z_{1} z_{2} \Phi\left(z_{1} z_{2}\right)+z_{1}^{2} z_{2}^{2} \varphi\left(z_{1} z_{2}\right) \Phi\left(z_{1} z_{2}\right)\left(1-q^{\lambda-\mu}\right)\right) . \\
\cdot \frac{\varphi_{\left(n_{1}+n_{2}\right) / 2+\lambda}\left(z_{1} z_{2}\right) / \Phi_{-\left(n_{1}+n_{2}\right) / 2-\mu}\left(q z_{1} z_{2}\right)}{z_{1}^{n_{1}} z_{2}^{n_{2}}} .
\end{aligned}
$$


THEOREM 23. With the assumptions of Theorem 22, the coefficients in the expansion

$$
g(z)=\sum_{k \in \mathbf{Z}^{2}} c_{k} \frac{z_{1}^{k_{1}} z_{2}^{k_{2}}}{\varphi_{k_{1}+\lambda}\left(q z_{1} z_{2}\right) / \Phi_{-k_{2}-\mu}\left(z_{1} z_{2}\right)}
$$

can be evaluated by

$$
\begin{aligned}
c_{n}=\left\langle z^{0}\right\rangle g(z)\left(1-z_{1} z_{2} \varphi\left(z_{1} z_{2}\right)-z_{1} z_{2} \Phi\left(z_{1} z_{2}\right)\right. \\
\left.+z_{1}^{2} z_{2}^{2} \varphi\left(z_{1} z_{2}\right) \Phi\left(z_{1} z_{2}\right)\left(1-q^{\lambda-\mu+n_{1}-n_{2}}\right)\right) \\
. \frac{\varphi_{n_{1}+\lambda}\left(z_{1} z_{2}\right) / \Phi_{-n_{2}-\mu}\left(q z_{1} z_{2}\right)}{z_{1}^{n_{1}} z_{2}^{n_{2}}} \cdot
\end{aligned}
$$

\section{REFERENCES}

1. R. A. Askey and M. E. H. Ismail, Recurrence relations, continued fractions and orthogonal polynomials, Mem. Amer. Math. Soc. 49 (1984), no. 300.

2. L. Carlitz, Some inverse relations, Duke Math. J. 40 (1973), 893-901.

3. J. Cigler, Operatormethoden für q-Identitäten, Monatsh. Math. 88 (1979), 87-105.

4. G. P. Egorychev, Integral representation and the computation of combinatorial sums, "Nauka" Sibirsk. Otdel., Novosibirsk, 1977; English transl., Transl. Math. Monographs, no. 59, Amer. Math. Soc., Providence, R. I., 1984.

5. L. Euler, De serie Lambertina plurimisque eius insignibus proprietatibus, Acta Acad. Sci. Petro, 1779; II, 1783, pp. 29-51; reprinted in Opera omnia, Ser. I, vol. 6, Teubner, Leipzig, 1921, pp. 350-369.

6. J. Fürlinger and J. Hofbauer, q-Catalan numbers, J. Combin. Theory A 40 (1985), 248-264.

7. A. M. Garsia, A q-analogue of the Lagrange inversion formula, Houston J. Math. 7 (1981), 205-237.

8. A. M. Garsia and S. A. Joni, A new expression for umbral operators and power series inversion, Proc. Amer. Math. Soc. 64 (1977), 179-185.

9. Algebra 6 (1978), 1187-1215.

10. I. Gessel, $A$ noncommutative generalization and $q$-analog of the Lagrange inversion formula, Trans. Amer. Math. Soc. 257 (1980), 455-482.

11. I. Gessel and D. Stanton, Applications of $q$-Lagrange inversion to basic hypergeometric series, Trans. Amer. Math. Soc. 277 (1983), 173-203.

12. __ Another family of $q$-Lagrange inversion formulas, Rocky Mountain J. Math. 16 (1986), 373-384.

13. I. J. Good, Generalization to several variables of Lagrange's expansion, with application to stochastic processes, Proc. Cambridge Philos. Soc. 56 (1960), 367-380.

14. H. W. Gould and L. C. Hsu, Some new inverse series relations, Duke Math. J. 40 (1973), 885-891.

15. J. Hofbauer, A q-analog of the Lagrange expansion, Arch. Math. 42 (1984), 536-544.

16. _ Lagrange-inversion (Seminaire Lotharingien de Combinatoire), I.R.M.A. ${ }^{\circ}$ 191/S-05, Strasbourg, 1982.

17. S. A. Joni, Lagrange inversion in higher dimensions and umbral operators, Linear and Multilinear Algebra 6 (1978), 111-121.

18. C. Krattenthaler, A new q-Lagrange formula and some applications, Proc. Amer. Math. Soc. 90 (1984), 338-344.

19. _ , q-Lagrangeformel und inverse Relationen, Ph.D. dissertation, Vienna, 1983.

20. P. A. MacMahon, Combinatory analysis, 2 vols., Cambridge Univ. Press, London, 1915-1916; reprint, Chelsea, New York, 1960.

21. _ Collected papers, Vol. I: Combinatorics, MIT Press, Cambridge, Mass., 1978.

22. J. Riordan, Combinatorial identities, Wiley, New York, 1968.

23. S. S. Abhyankar, Lectures in algebraic geometry, Notes by Chris Christensen, Purdue Univ., 1974. 
24. A. Brini, Higher dimensional recursive matrices and diagonal delta sets of series, J. Combin. Theory Ser. A 36 (1984), 315-331.

25. A. M. Garsia and S. A. Joni, Composition sequences, Comm. Algebra 6 (1978), 1187-1215.

26. I. Gessel, A combinatorial proof of the multivariable Lagrange inversion formula, J. Combin. Theory Ser. A 45 (1987), 178-196.

27. P. Henrici, Die Lagrange-Bürmannsche Formel bei formalen Potenzreihen, Jber. Deutsch Math.Verein 86 (1984), 115-134.

28. O. Viskov, Inversion of power series and the Lagrange formula, Soviet Math. Dokl. 22 (1980), 330-332.

INSTITUT FÜR MATHEMATIK DER UNIVERSitÄt Wien 1090, STRUdlhofgasse 4, WiEN, AUSTRIA 